\title{
Acoustic Pressure Waves in Vibrating 3-D Laminated Beam-Plate Enclosures
}

\author{
Charles A. Osheku, ${ }^{1}$ Vincent O. S. Olunloyo, ${ }^{1}$ O. Damisa, ${ }^{2}$ and Theddeus T. Akano ${ }^{1}$ \\ ${ }^{1}$ Department of Systems Engineering, Faculty of Engineering, University of Lagos, Akoka-Yaba, Lagos 23401, Nigeria \\ ${ }^{2}$ Department of Mechanical Engineering, Faculty of Engineering, University of Lagos, Nigeria
}

Correspondence should be addressed to Charles A. Osheku, charlesosheku2002@yahoo.com

Received 22 September 2009; Accepted 5 December 2009

Recommended by Abul Azad

The effect of structural vibration on the propagation of acoustic pressure waves through a cantilevered 3-D laminated beam-plate enclosure is investigated analytically. For this problem, a set of well-posed partial differential equations governing the vibroacoustic wave interaction phenomenon are formulated and matched for the various vibrating boundary surfaces. By employing integral transforms, a closed form analytical expression is computed suitable for vibroacoustic modeling, design analysis, and general aerospace defensive applications. The closed-form expression takes the form of a kernel of polynomials for acoustic pressure waves showing the influence of linear interface pressure variation across the axes of vibrating boundary surfaces. Simulated results demonstrate how the mode shapes and the associated natural frequencies can be easily computed. It is shown in this paper that acoustic pressure waves propagation are dynamically stable through laminated enclosures with progressive decrement in interfacial pressure distribution under the influence of high excitation frequencies irrespective of whether the induced flow is subsonic, sonic , supersonic, or hypersonic. Hence, in practice, dynamic stability of hypersonic aircrafts or jet airplanes can be further enhanced by replacing their noise transmission systems with laminated enclosures.

Copyright (C) 2009 Charles A. Osheku et al. This is an open access article distributed under the Creative Commons Attribution License, which permits unrestricted use, distribution, and reproduction in any medium, provided the original work is properly cited.

\section{Introduction}

The control of vibration and noise propagation from industrial plants, aircraft engines and noise generating machines has remained an active research area for several decades. Nonetheless, limited literature exist in the area of noisestructure dynamic interaction modeling. However, within the context of analytical and experimental studies in acoustic -structure dynamics, a number of investigations have been reported in [1-9]. For these problems, analytical techniques were employed to study active control of acoustic-structure interaction in 2-D and 3-D enclosures.

In particular, Fang et al. [10], investigated acousticstructure interaction through a 3-D rectangular enclosure to improve control design analysis. Nevertheless, reliable low-order finite model of 3-D acoustic-structure interaction has posed a formidable challenge in the field, given the fact that model density and order increase from 1-D to 3D configurations, especially for severe multiple input and output systems. In the field of acoustic-structure interaction, the scarcity of literature in analytical modeling of 3-D enclosures having complex boundary conditions has been attributed mainly to the intractability of dynamic equations which preclude closed form solutions.

For some cases, with simplified assumptions, closedform solutions are possible usually with rigorous mathematical intrigues and manipulations. One of such methods involves the computation of a transfer function from the frequency response data. Even at that, the limit of these identification techniques is that, the system identification algorithms work satisfactorily only for low-order systems and systems with separated nodes.

However, for 3-D acoustic enclosures, with vibrating boundary surfaces, these identification techniques are fronted with difficulty, especially with two-input one-output systems. Nevertheless, investigations of vibroacoustic pressure waves propagation through a 3-D acoustic enclosure having vibrating laminated boundary surfaces has not been 
widely reported in literature. Within the context of transverse vibrations of laminated structures, with non-uniform interfacial pressure distribution, some interesting results have been reported recently in [11-15]. The effect of structural vibration on the propagation of acoustic pressure waves through a cantilevered 3-D laminated beam-plate enclosure is investigated. For this problem, a set of well posed partial differential equations governing the vibroacoustic pressure waves interaction phenomenon are formulated and matched for the various vibrating boundary surfaces. This paper is organized as follows. Section 1 introduces the problem under investigation within a general context. In the next section, the essential analytical mechanics is briefly reviewed. In Section 3, these relationships are incorporated into a simplified analytical model for the mathematical analysis of the vibroacoustic pressure waves problem. Section 4 is concerned with the analysis of the transmission intensity as modulated by interfacial pressure variation and excitation frequency.

In Section 5, simulated results for the vibroacoustic pressure waves, as influenced by the linear interface pressure variations across the axes of the vibrating boundary surfaces are analyzed whilst in Section 6, the paper ends with the summary and conclusion.

\section{Problem Formulation}

The problem here is to examine analytically, the effect of the nature of load, frequency variation and the pressure gradient on the acoustic pressure waves propagation.

A general theory of the energy dissipation properties of press-fit joints in the presence of Coulomb friction as originally developed by Goodman and Klumpp provides the basis for the physics of the problem. As illustrated in Figure 1(a) below the proposed 3-D enclosures is constructed with laminated elastic structure for each boundary surface. The contact conditions between the mating layers as itemised in Damisa et al. [11] hold, namely,

(i) there is continuity of stress distributions at the interface to sufficiently hold the equivalent layers together both in the pre- and post-slip conditions,

(ii) a stick elastic slip with presence of Coulomb friction occurs at the interface of the sandwich vibrating boundary to dissipate energy and does not remain constant as a function of some other variables such as spatial distance, time or velocity.

The formulated vibroacoustic wave equation is given by the relation

$$
\nabla^{2} P-\frac{1 \partial^{2} P}{c_{1}^{2} \partial t^{2}}-\frac{2 u_{x} \partial^{2} P}{c_{1}^{2} \partial t \partial x}-\frac{u_{x}^{2}}{c_{1}^{2}} \frac{\partial^{2} P}{\partial x^{2}}=-\frac{1 \partial^{2} W_{1}}{\rho \partial t^{2}}
$$

while the governing equations for each vibrating boundaries $\Omega_{1}, \Omega_{2}, \Omega_{3}, \Omega_{4}$ are derived in the appendix. In the meantime, the 2-D views of the structural loadings through $\Omega_{3}, \Omega_{4}$ are illustrated in Figures (1(b) and 1(c)) for brevity.

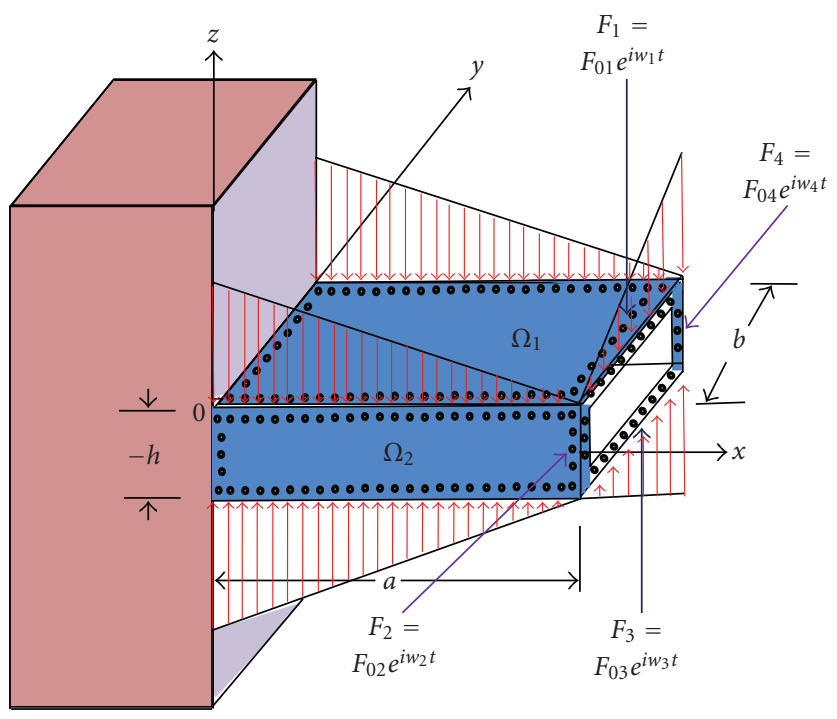

(a) Problem geometry of 3-D composite structure.

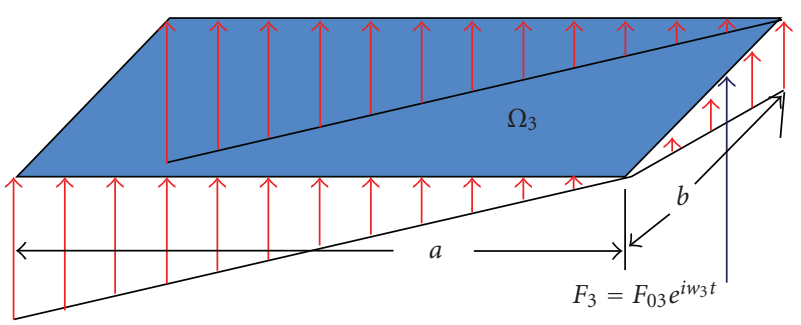

(b) 2-D view of the composite structure geometry through $\Omega_{3}$

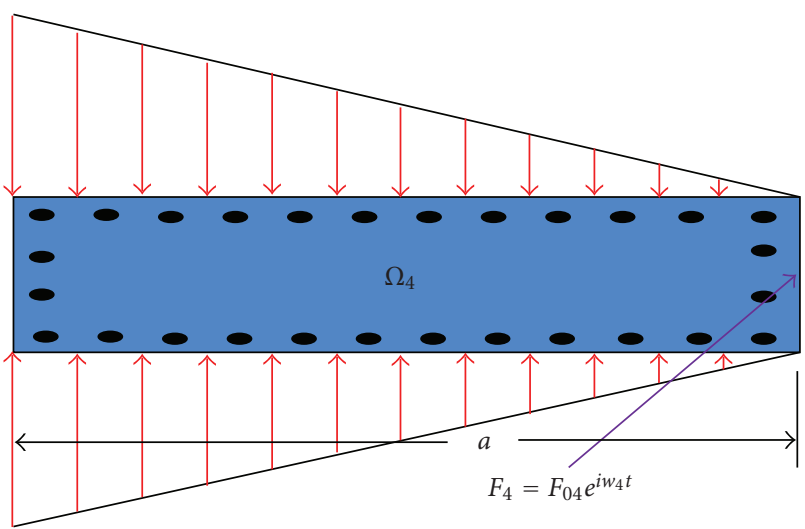

(c) 2-D view of the composite structure geometry through $\Omega_{4}$

Figure 1:

\section{Mathematical Analysis of the Vibroacoustic Pressure Waves Equation}

In this investigation we will simplify the solution of (1) by assuming that the prompting vibrating membrane is through domain $\Omega_{1}$ while the boundary effects on the pressure waves propagation are restricted to the following domains $\left(\Omega_{2}, \Omega_{3}\right.$, $\left.\Omega_{4}\right)$. 


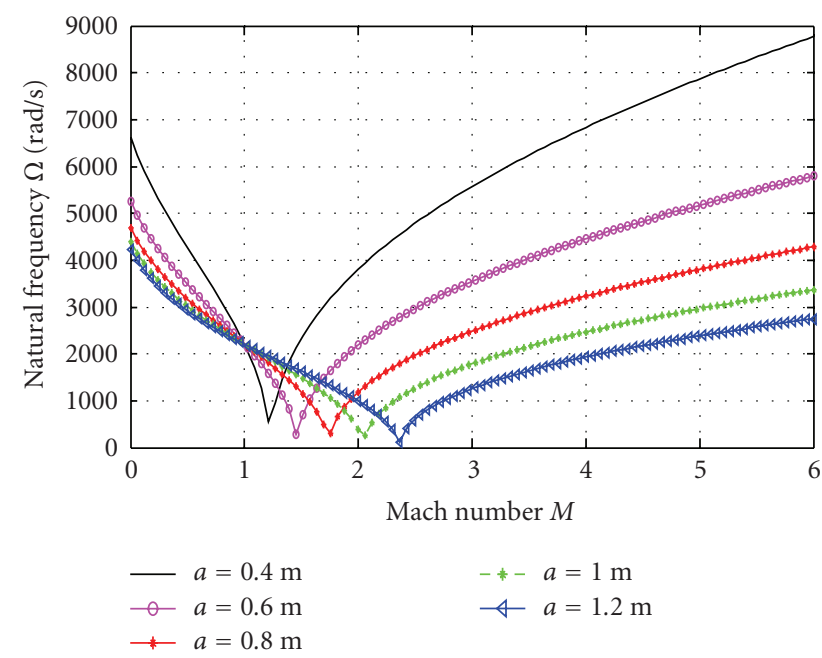

Figure 2: Acoustic natural frequency profile for the case $m=1 ; n=$ $1 ; k=1 ; b=0.3 ; c=0.8$.

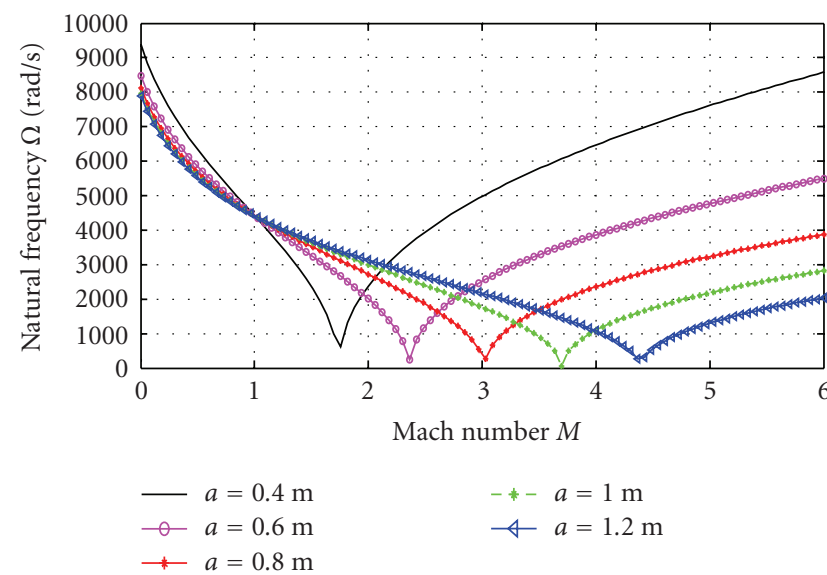

FIGURE 3: Acoustic natural frequency profile for the case $m=2 ; n=$ $2 ; k=2 ; b=0.3 ; c=0.8$.

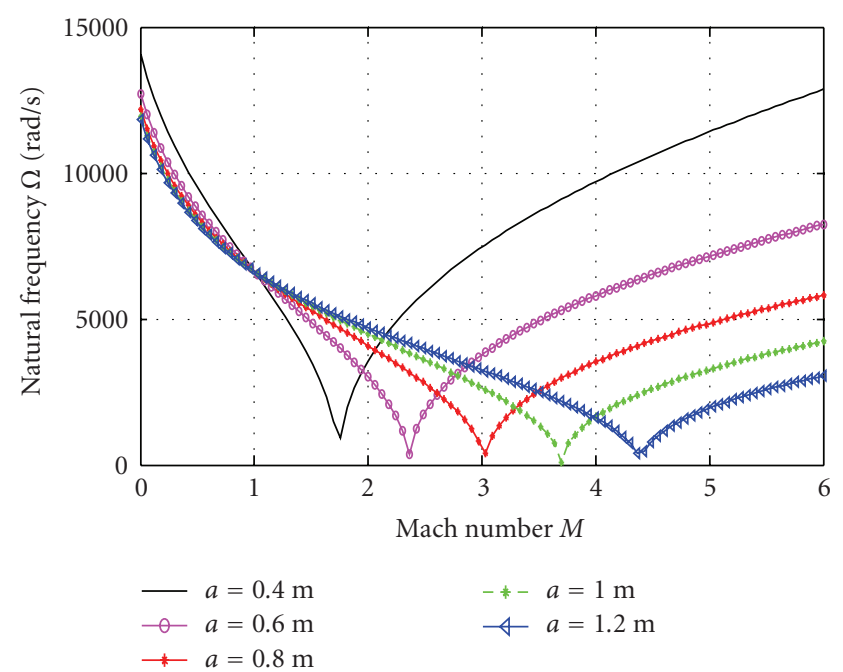

FIGURE 4: Acoustic natural frequency profile for the case $m=3 ; n=$ $3 ; k=3 ; b=0.3 ; c=0.8$.

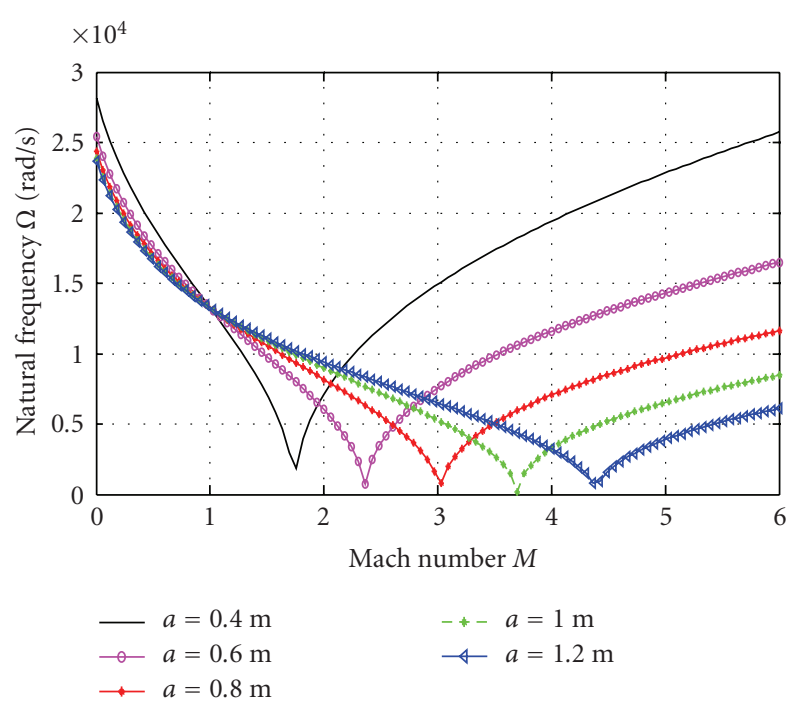

FIGURE 5: Acoustic natural frequency profile for the case $m=6 ; n=$ $6 ; k=6 ; b=0.3 ; c=0.8$.

Under this circumstance, (1) takes the form, viz,

$$
\begin{gathered}
\nabla^{2} P-\frac{1}{c_{0}^{2}} \frac{\partial^{2} P}{\partial t^{2}}-\frac{2 M}{c_{0}} \frac{\partial^{2} P}{\partial t \partial x}-M^{2} \frac{\partial^{2} P}{\partial x^{2}}=-\frac{1}{\rho} \frac{\partial^{2} W_{1}}{\partial t^{2}}, \\
\forall M=\frac{u_{x}}{c_{0}} .
\end{gathered}
$$

Equation (2) can be further expressed as

$$
\left(1-M^{2}\right) \frac{\partial^{2} P}{\partial x^{2}}+\frac{\partial^{2} P}{\partial y^{2}}+\frac{\partial^{2} P}{\partial z^{2}}-\left(\frac{1+2 M}{c_{0}^{2}}\right) \frac{\partial^{2} P}{\partial t^{2}}=-\frac{1}{\rho} \frac{\partial^{2} W_{1}}{\partial t^{2}} .
$$

By introducing the triple Fourier Finite Sine Transform, namely

$$
\begin{aligned}
\operatorname{FFF}_{\mathcal{S}}\{[\bullet]\} & =[\bar{\bullet}] \\
& =\int_{0}^{b} \int_{0}^{a} \int_{0}^{c}[\bullet] \mathfrak{X} d x d y d z, \\
{[\bullet] } & =\frac{8}{a b c} \sum_{k=1}^{\infty} \sum_{m=1}^{\infty} \sum_{n=1}^{\infty}[\bar{\bullet}] \mathfrak{X},
\end{aligned}
$$

where $\mathfrak{X}$ denotes $\sin (n \pi x / a) \sin (m \pi y / b) \sin (k \pi z / c)$, in conjunction with the Laplace transform, viz,

$$
(\widetilde{\bullet})=\int_{0}^{\infty}(\bullet) e^{-s t} d t, \quad(\bullet)=\frac{1}{2 \pi i} \int_{\eta-i \infty}^{\eta+i \infty}(\widetilde{\bullet}) e^{s t} d s .
$$


Equation (3) in the transform plane, subject to zero initial conditions takes the following form, viz,

$$
\begin{aligned}
& \left(1-M^{2}\right)\left(-\frac{n^{2} \pi^{2}}{a^{2}} \widetilde{P}^{F_{x} F_{y} F_{z}}\left(\lambda_{n}, \lambda_{m}, \lambda_{k}, s\right)\right. \\
& +\frac{n \pi}{a}(-1)^{n+1} \widetilde{P}^{F_{y} F_{z}}\left(a, \lambda_{m}, \lambda_{k}, s\right) \\
& \left.+\frac{n \pi}{a} \widetilde{P}^{F_{y} F_{z}}\left(0, \lambda_{m}, \lambda_{k}, s\right)\right) \\
& +\left(-\frac{m^{2} \pi^{2}}{b^{2}} \widetilde{P}^{F_{x} F_{y} F_{z}}\left(\lambda_{n}, \lambda_{m}, \lambda_{k}, s\right)\right. \\
& +\frac{m \pi}{b}(-1)^{m} \widetilde{P}^{F_{x} F_{z}}\left(\lambda_{m}, b, \lambda_{k}, s\right) \\
& \left.+\frac{m \pi}{b} \widetilde{P}^{F_{x} F_{z}}\left(\lambda_{m}, 0, \lambda_{k}, s\right)\right) \\
& +\left(-\frac{k^{2} \pi^{2}}{c^{2}} \widetilde{P}^{F_{x} F_{y} F_{z}}\left(\lambda_{n}, \lambda_{m}, \lambda_{k}, s\right)\right. \\
& +\frac{k \pi}{c}(-1)^{k+1} \widetilde{P}^{F_{x} F_{y}}\left(\lambda_{n}, \lambda_{m}, c, s\right) \\
& \left.+\frac{k \pi}{c} \widetilde{P}^{F_{x} F_{y}}\left(\lambda_{n}, \lambda_{m}, 0, s\right)\right) \\
& -\left(\frac{1+2 M}{c_{0}^{2}}\right) s^{2} \widetilde{P}^{F_{x} F_{y} F_{z}}\left(\lambda_{n}, \lambda_{m}, \lambda_{k}, s\right) \\
& =\frac{1}{\rho} s^{2} W_{1} F_{x} F_{y} F_{z}\left(\lambda_{n}, \lambda_{m}, \lambda_{k}, s\right),
\end{aligned}
$$

where the following have been defined

$$
\begin{aligned}
& \widetilde{P}^{F_{x}} F_{y} F_{z}\left(\lambda_{n}, \lambda_{m}, \lambda_{k}, s\right) \\
& =-\int_{0}^{b} \int_{0}^{a} \int_{0}^{c}[\tilde{P}] \sin \left(\frac{n \pi x}{a}\right) \sin \left(\frac{m \pi y}{b}\right) \sin \left(\frac{k \pi z}{c}\right) d x d y d z \\
& \tilde{P}^{F_{y}} F_{z}\left(a, \lambda_{m}, \lambda_{k}, s\right) \\
& =-\int_{0}^{b} \int_{0}^{c}[\tilde{P}(a, y, z, s)] \sin \left(\frac{m \pi y}{b}\right) \sin \left(\frac{k \pi z}{c}\right) d y d z \\
& \widetilde{P}^{F_{y} F_{z}}\left(0, \lambda_{m}, \lambda_{k}, s\right) \\
& =-\int_{0}^{b} \int_{0}^{c}[\tilde{P}(0, y, z, s)] \sin \left(\frac{m \pi y}{b}\right) \sin \left(\frac{k \pi z}{c}\right) d y d z \\
& \widetilde{P}^{F_{x}} F_{z}\left(\lambda_{n}, 0, \lambda_{k}, s\right) \\
& =-\int_{0}^{a} \int_{0}^{c}[\tilde{P}(x, 0, z, s)] \sin \left(\frac{n \pi x}{a}\right) \sin \left(\frac{k \pi z}{c}\right) d y d z \\
& \widetilde{P}^{F_{x}} F_{z}\left(\lambda_{n}, b, \lambda_{k}, s\right) \\
& =-\int_{0}^{b} \int_{0}^{c}[\tilde{P}(x, b, z, s)] \sin \left(\frac{n \pi x}{a}\right) \sin \left(\frac{k \pi z}{c}\right) d y d z .
\end{aligned}
$$

Next we can evaluate the boundary stresses through the domains $\Omega_{2}, \Omega_{3}, \Omega_{4}$ from the following relations, viz,

$$
\begin{aligned}
& \widetilde{P}^{F_{y} F_{z}}\left(0, \lambda_{m}, \lambda_{k}, s\right) \\
& =\int_{0}^{b} \int_{0}^{c}\left[\mu P_{\mathrm{av}}\right] \sin \left(\frac{m \pi y}{b}\right) \sin \left(\frac{k \pi z}{c}\right) d y d z, \\
& \widetilde{P}^{F_{y} F_{z}}\left(a, \lambda_{m}, \lambda_{k}, s\right) \\
& =\int_{0}^{b} \int_{0}^{c}\left[\mu P_{\mathrm{av}}\right] \sin \left(\frac{m \pi y}{b}\right) \sin \left(\frac{k \pi z}{c}\right) d y d z, \\
& \widetilde{P}^{F_{x} F_{y}}\left(\lambda_{n}, \lambda_{m}, 0, s\right) \\
& =\int_{0}^{b} \int_{0}^{a}\left[\frac{P_{\mathrm{av}}}{s}\right] \sin \left(\frac{n \pi x}{a}\right) \sin \left(\frac{m \pi y}{b}\right) d x d y, \\
& \widetilde{P}^{F_{x} F_{y}}\left(\lambda_{n}, \lambda_{m}, c, s\right) \\
& =-\int_{0}^{b} \int_{0}^{a}\left[\frac{P_{\mathrm{av}}}{s}\right] \sin \left(\frac{m \pi x}{a}\right) \sin \left(\frac{k \pi y}{b}\right) d x d y, \\
& \widetilde{P}^{F_{x} F_{z}}\left(\lambda_{n}, b, \lambda_{k}, s\right) \\
& =\int_{0}^{a} \int_{0}^{c}\left[\frac{P_{\mathrm{av}}}{s}\right] \sin \left(\frac{n \pi x}{a}\right) \sin \left(\frac{k \pi z}{c}\right) d x d z, \\
& \widetilde{P}^{F_{x}} F_{z}\left(\lambda_{n}, 0, \lambda_{k}, s\right) \\
& =-\int_{0}^{c}\left[\frac{P_{\mathrm{av}}}{s}\right] \sin \left(\frac{n \pi x}{a}\right) \sin \left(\frac{k \pi z}{c}\right) d x d z .
\end{aligned}
$$

So that on appropriate substitution, (6) is now expressed in Fourier-Laplace transform plane as

$$
\begin{aligned}
(1- & \left.M^{2}\right)\left(-\frac{n^{2} \pi^{2}}{a^{2}} \widetilde{P}^{F_{x} F_{y} F_{z}}\left(\lambda_{n}, \lambda_{m}, \lambda_{k}, s\right)\right. \\
& \left.+\mathfrak{k}\left(\left(\frac{\left(1+(-1)^{m+1}\right)}{m \pi}\right)\left(\frac{\left(1+(-1)^{k+1}\right)}{k \pi}\right)\right)\right) \\
& -\frac{m^{2} \pi^{2}}{b^{2}} \widetilde{P}^{F_{x} F_{y} F_{z}}\left(\lambda_{n}, \lambda_{m}, \lambda_{k}, s\right) \\
+ & \left(\frac{m \pi}{b}(-1)^{m+1}+\frac{m \pi}{b}\right) \frac{\mu P_{\mathrm{av}}(0, b)}{s}(b) \\
& -\frac{k^{2} \pi^{2}}{c^{2}} \widetilde{P}^{F_{x} F_{y} F_{z}}\left(\lambda_{n}, \lambda_{m}, \lambda_{k}, s\right) \\
& +\left(\frac{k \pi}{c}(-1)^{k+1}+\frac{k \pi}{c}\right) \frac{P_{\mathrm{av}}(a, b)}{s}(\mathcal{N}) \\
& -\left(\frac{1+2 M}{c_{0}^{2}}\right) s^{2} \widetilde{P}^{F_{x} F_{y} F_{z}}\left(\lambda_{n}, \lambda_{m}, \lambda_{k}, s\right) \\
= & \frac{1}{\rho} s^{2} W_{1} F_{x} F_{y} F_{z}\left(\lambda_{n}, \lambda_{m}, \lambda_{k}, s\right),
\end{aligned}
$$

where $\mathfrak{k}$ denotes $\left(n \pi / a(-1)^{n+1}+n \pi / a\right) \mu P_{\mathrm{av}}(a, 0) / s, \quad b$ denotes $\left(\left(1+(-1)^{m+1}\right) / m \pi\right)\left(\left(1+(-1)^{k+1}\right) / k \pi\right), \mathcal{N}$ denotes 
$\left(\left(1+(-1)^{n+1}\right) / n \pi\right)\left(\left(1+(-1)^{m+1}\right) / m \pi\right)$. Subject to the following pressure profiles prescribed in Olunloyo et al. [12], viz,

$$
\begin{aligned}
& p(x, 0)=p_{0}\left(1+\psi_{1} \frac{x}{a}\right), \\
& p(0, y)=p_{0}\left(1+\psi_{2} \frac{y}{b}\right),
\end{aligned}
$$

This now allows us to obtain the vibroacoustic pressure in the transform plane as

$$
\begin{aligned}
& \widetilde{P}^{F_{x} F_{y} F_{z}}\left(\lambda_{n}, \lambda_{m}, \lambda_{k}, s\right) \\
& =c_{0}^{2}\left(( 1 - M ^ { 2 } ) \left(\left(\frac{n \pi}{a}(-1)^{n+1}+\frac{n \pi}{a}\right)\right.\right. \\
& \times \frac{\mu P_{\mathrm{av}}(a, 0)}{s} \\
& \left.\times\left(b c\left(\frac{\left(1+(-1)^{m+1}\right)}{m \pi}\right)\left(\frac{\left(1+(-1)^{k+1}\right)}{k \pi}\right)\right)\right) \\
& +\left(\frac{m \pi}{b}(-1)^{m+1}+\frac{m \pi}{b}\right) \frac{\mu P_{\mathrm{av}}(0, b)}{s} \\
& \times\left(b c\left(\frac{\left(1+(-1)^{m+1}\right)}{m \pi}\right)\left(\frac{\left(1+(-1)^{k+1}\right)}{k \pi}\right)\right) \\
& +\frac{k \pi}{c}\left((-1)^{k}+1\right) \frac{P_{\mathrm{av}}(a, b)}{s} \\
& \times\left(a b\left(\frac{\left(1+(-1)^{n+1}\right)}{n \pi}\right)\left(\frac{\left(1+(-1)^{m+1}\right)}{m \pi}\right)\right) \\
& \left.+\left(\frac{1}{\rho}\right) s^{2} W_{1} F_{x} F_{y} F_{z}\left(\lambda_{n}, \lambda_{m}, \lambda_{k}, s\right)\right) /(1+2 M) \\
& \times\left(s^{2}+\left(\frac{c_{0}^{2}}{1+2 M}\right)\left(\left(1-M^{2}\right) \frac{n^{2} \pi^{2}}{a^{2}}+\frac{m^{2} \pi^{2}}{b^{2}}+\frac{k^{2} \pi^{2}}{c^{2}}\right)\right),
\end{aligned}
$$

$$
\forall P_{\mathrm{av}}(a, 0)=P_{0}\left(1+\frac{\psi_{1}}{2}\right) a,
$$$$
P_{\mathrm{av}}(0, b)=P_{0}\left(1+\frac{\psi_{2}}{2}\right) b \text {, }
$$$$
P_{\mathrm{av}}(a, b)
$$

$$
\begin{array}{r}
=P_{0} a \sqrt{\left(\left(\frac{\left(1+\left(\psi_{1} / 2\right)\right)^{2}}{\left(1+r^{2}\right)}\right)+\left(\frac{\left(r\left(1+\left(\psi_{1} / 2\right)\right)\right)^{2}}{\left(1+r^{2}\right)}\right)\right)}, \\
r=\frac{b}{a} .
\end{array}
$$

To complete the solution of (10), we have employed the following double Fourier sine transform relations, namely,

$$
\begin{aligned}
& F F_{S}\left\{\left[\widetilde{W}_{x x x x}\right]\right\}=\frac{m^{4} \pi^{4}}{a^{4}} \widetilde{W}^{F_{x} F_{y}}\left(s, \lambda_{m}, \lambda_{n}\right) \\
& -\frac{m^{3} \pi^{3}}{a^{3}}\left\{\widetilde{W}^{F_{y}}\left(s, 0, \lambda_{n}\right)+\right. \\
& \left.(-1)^{m+1} \widetilde{W}\left(s, 0, \lambda_{n}\right)\right\} \\
& +\frac{m \pi}{a}\left\{\widetilde{W}_{x x}^{F_{y}}\left(s, 0, \lambda_{n}\right)+\right. \\
& \left.(-1)^{m+1} \widetilde{W}_{x x}\left(s, a, \lambda_{n}\right)\right\} \\
& F F_{S}\left\{\widetilde{W}_{x x y y}(s, x, y)\right\}=\frac{m^{2} n^{2} \pi^{4}}{a^{2} b^{2}} \widetilde{W}^{F_{x} F_{y}}\left(s, \lambda_{m}, \lambda_{n}\right) \\
& -\frac{m^{2} n \pi^{3}}{a^{2} b}\left\{\widetilde{W}^{F_{y}}\left(s, \lambda_{m}, 0\right)\right. \\
& \left.+(-1)^{n+1} \widetilde{W}\left(s, \lambda_{m} b\right)\right\} \\
& -\frac{m n^{2} \pi^{3}}{a b^{2}}\left\{\widetilde{W}^{F_{\bar{y}}}\left(s, 0, \lambda_{n}\right)\right. \\
& \left.+(-1)^{m+1} \widetilde{W}\left(s, a, \lambda_{n}\right)\right\} \\
& +\frac{m n \pi^{2}}{a b}(-1)^{n+1}\{\widetilde{W}(s, 0,0) \\
& \left.+(-1)^{m+1} \widetilde{W}(s, 0, b)\right\} \\
& +(-1)^{m} \frac{m n \pi^{2}}{a b}\{\widetilde{W}(s, 0,0) \\
& \left.+(-1)^{m+1} \widetilde{W}(s, 0, b)\right\} \\
& F F_{S}\left\{\widetilde{W}_{y y y y}(s, x, y)\right\}=\frac{n^{4} \pi^{4}}{b^{4}} \widetilde{W}^{F_{y} F_{x}}\left(s, \lambda_{n}, \lambda_{m}\right) \\
& -\frac{n^{3} \pi^{3}}{b^{3}}\left\{\widetilde{W}^{F_{x}}\left(s, \lambda_{m}, 0\right)\right. \\
& \left.+(-1)^{n+1} \widetilde{W}\left(s, \lambda_{m}, 0\right)\right\} \\
& +\frac{n \pi}{b}\left\{\widetilde{W}_{y y}^{F_{x}}\left(s, \lambda_{m}, 0\right)\right. \\
& \left.+(-1)^{n+1} \widetilde{W}_{y y}\left(s, \lambda_{m}, b\right)\right\} .
\end{aligned}
$$

By employing (11), (13), and (14) subject to (A.5), (A.6), (A.7), (A.8), (A.9), and (A.10), $W_{1} F_{x} F_{y} F_{z}\left(\lambda_{n}, \lambda_{m}, \lambda_{k}, s\right)$ can be evaluated from (A.1) as

$$
\begin{aligned}
& W_{1}^{F}\left(\lambda_{n}, \lambda_{m}, \lambda_{k}, s\right) \\
& =\left(c \left(\left(\left(\frac{m \pi}{a}+\frac{n \pi}{b}\left(\frac{-r^{2}}{v}\right)\right)\left(\mathfrak{w}-\frac{6 \mu p_{0}\left(1+\left(\psi_{1} / 2\right)\right)}{s E H_{z}^{2}}\right)\right.\right.\right. \\
& \left.\quad \times a+\left(\frac{\mu H_{z}}{2}\right)\left(\frac{p_{0}}{a} \psi_{1}+\frac{p_{0}}{b} \psi_{2}\right)\right) / \rho H_{z}\left(s^{2}+\frac{D}{\rho H_{z}}\right. \\
& \left.\left.\left.\quad \times\left(\frac{n^{4} \pi^{4}}{a^{4}}+2 \frac{n^{2} m^{2} \pi^{4}}{a^{2} b^{2}}+\frac{m^{4} \pi^{4}}{b^{4}}\right)\right)\right)\right)(\mathfrak{S}),
\end{aligned}
$$


where $\mathfrak{w}$ denotes $6 \tilde{F} / E b H_{z}{ }^{3}$, and $\mathfrak{S}$ denotes $\left(1+(-1)^{k+1}\right) / k \pi$. So that on appropriate substitution into (10), the vibroacoustic pressure can be evaluated in the transform plane as

$$
\begin{aligned}
\widetilde{P}^{F}\left(s, \lambda_{n}, \lambda_{m}, \lambda_{k}\right) \\
=c_{0}^{2}\left(\left(1-M^{2}\right) \chi_{1}(m, n, k) \mu \rho_{0}\left(1+\frac{\psi_{1}}{2}\right)\right. \\
\quad+\chi_{2}(m, n, k) \mu \rho_{0}\left(1+\frac{\psi_{1}}{2}\right)+\chi_{3}(m, n, k) \mu \rho_{0} \Psi \\
\quad+c\left(\chi_{4}(m, n, k)\left(\frac{6 F_{01}}{E b H_{3}^{3}(s-i \omega)}-\frac{6 \mu \rho_{0}\left(1+\psi_{2} / 2\right)}{s E H_{z}^{2}}\right)\right. \\
\left.\left.\quad+\chi_{5}(m, n, k) \mu r^{-1} b H_{z} \rho_{0}\left(\psi_{1}+r^{-1} \psi_{2}\right)\right) / \mathfrak{z}\right) \\
/(1+2 M)\left(s^{2}+\Omega^{2}\right)\left(1+2\left(\frac{n}{m}\right)^{2} r^{2}+\left(\frac{n}{m}\right)^{4} r^{4}\right) \omega_{0}^{-2},
\end{aligned}
$$

where $\mathfrak{z}$ denotes $\rho^{2}\left(s^{2}+\omega_{0}^{2}\right)$, where the following have been defined, viz,

$$
\begin{aligned}
& \chi_{1}(m, n, k) \\
& =\frac{(n / m)\left(\rho H_{z} b c / a^{4} D\right)\left(\mathfrak{A}\left(1+(-1)^{k+1} / k \pi\right)\right)}{\left(1+2(n / m)^{2} r^{2}+(n / m)^{4} r^{4}\right)}, \\
& \chi_{2}(m, n, k) \\
& =\frac{a^{4} b c(n / m)\left(1+(-1)^{m+1} / m^{3} \pi^{3}\right) \mathfrak{B}}{\left(1+2(n / m)^{2} r^{2}+(n / m)^{4} r^{4}\right)}, \\
& \chi_{3}(m, n, k) \\
& =\frac{a b / c(k / m)^{2}\left(1+(-1)^{m+1} / m^{3} \pi^{3}\right) \mathfrak{C}}{\left(1+2(n / m)^{2} r^{2}+(n / m)^{4} r^{4}\right)} \\
& \chi_{4}(m, n, k) \\
& =\frac{a^{4}\left(\mathfrak{D}\left(1+(-1)^{k+1} / k \pi\right)\right)}{\left(1+2(n / m)^{2} r^{2}+(n / m)^{4} r^{4}\right)}, \\
& \chi_{5}(m, n, k) \\
& =\left(\frac{n}{m}\right) \frac{(\mathfrak{E})}{\left(1+2(n / m)^{2} r^{2}+(n / m)^{4} r^{4}\right)} \\
& \Psi=\sqrt{\left(\left(\frac{\mathfrak{F}^{2}}{\left(1+r^{2}\right)}\right)+\left(\frac{(\mathfrak{G})^{2}}{\left(1+r^{2}\right)}\right)\right)}
\end{aligned}
$$

where $\mathfrak{A}$ denotes $\left(1+(-1)^{m+1} / m^{3} \pi^{3}\right)\left(1+(-1)^{n+1} / n \pi\right), \mathfrak{B}$ denotes $(2 / n \pi)\left(1+(-1)^{k+1} / k \pi\right), \mathfrak{C}\left(1+(-1)^{n+1} / n \pi\right)(1+$ $\left.(-1)^{k+1} / k \pi\right), \mathfrak{D}$ denotes $\left(1-(n / m) r^{3} / v^{2}\right)\left(1 / m^{3} \pi^{3}\right)$, $\mathfrak{E}$ denotes $\left(1+(-1)^{k+1} / k \pi\right)\left(a^{4} / m^{3} \pi^{3}\right)(1 / n \pi), \mathfrak{F}$ denotes $\left(1+\left(\psi_{1} / 2\right)\right), \mathfrak{G}$ denotes $r\left(1+\left(\psi_{1} / 2\right)\right)$.
By invoking the Fourier inversion, the solution of (16) in the Laplace transform plane can be written as

$$
\widetilde{P}(x, y, z)=\frac{8}{a b c} \sum_{k=1}^{\infty} \sum_{n=1}^{\infty} \sum_{m=1}^{\infty}\left(\widetilde{P}^{F}\left(\lambda_{m}, \lambda_{n}, \lambda_{k}\right) \mathfrak{H}\right),
$$

where $\mathfrak{H}$ denotes $\sin (m \pi x / a) \sin (n \pi y / b) \sin (k \pi z / c)$.

The above equation can be simplified via the following relations, viz,

$$
\begin{aligned}
& \sum_{k=1}^{\infty} \sum_{n=1}^{\infty} \sum_{m=1}^{\infty} \chi_{1}(m, n, k) \mathfrak{I} \\
& =\sum_{k=1}^{\infty} \sum_{n=1}^{\infty} \sum_{m=1}^{\infty}\left(\frac{(n / m)\left(\rho H_{z} b c / a^{4} D\right) \mathfrak{L}}{\left(1+2(n / m)^{2} r^{2}+(n / m)^{4} r^{4}\right)}\right) \mathfrak{I},
\end{aligned}
$$

where $\mathfrak{I}$ denotes $\sin (m \pi x / a) \sin (n \pi y / b) \sin (k \pi z / c), \mathfrak{L}$ denotes $\left(\left(1+(-1)^{m+1} / m^{3} \pi^{3}\right)\left(1+(-1)^{n+1} / n \pi\right)\left(1+(-1)^{k+1} / k \pi\right)\right)$.

$$
\begin{aligned}
& \sum_{k=1}^{\infty} \sum_{n=1}^{\infty} \sum_{m=1}^{\infty} \chi_{2}(m, n, k) \mathfrak{f} \\
& =\sum_{k=1}^{\infty} \sum_{n=1}^{\infty} \sum_{m=1}^{\infty}\left(\frac{a^{4} b c(n / m) \mathfrak{h}}{\left(1+2(n / m)^{2} r^{2}+(n / m)^{4} r^{4}\right)}\right) \mathfrak{f}
\end{aligned}
$$

where $\mathfrak{f}$ denotes $\sin (m \pi x / a) \sin (n \pi y / b) \sin (k \pi z / c), \mathfrak{h}$ denotes $\left(1+(-1)^{m+1} / m^{3} \pi^{3}\right)(2 / n \pi)\left(1+(-1)^{k+1} / k \pi\right)$ :

$$
\begin{aligned}
& \sum_{k=1}^{\infty} \sum_{n=1}^{\infty} \sum_{m=1}^{\infty} \chi_{3}(m, n, k) \mathcal{Z} \\
& =\sum_{k=1}^{\infty} \sum_{n=1}^{\infty} \sum_{m=1}^{\infty}\left(\frac{a b / c(k / m)^{2}\left(1+(-1)^{m+1} / m^{3} \pi^{3}\right) \mathfrak{Z}}{\left(1+2(n / m)^{2} r^{2}+(n / m)^{4} r^{4}\right)}\right) \mathcal{Z},
\end{aligned}
$$

where $\mathcal{Z}$ denotes $\sin (m \pi x / a) \sin (n \pi y / b) \sin (k \pi z / c), \mathfrak{Z}$ denotes $\left(1+(-1)^{n+1} / n \pi\right)\left(1+(-1)^{k+1} / k \pi\right)$ :

$$
\begin{aligned}
& \sum_{k=1}^{\infty} \sum_{n=1}^{\infty} \sum_{m=1}^{\infty} \chi_{4}(m, n, k) \Re \\
& =\sum_{k=1}^{\infty} \sum_{n=1}^{\infty} \sum_{m=1}^{\infty}\left(\frac{\pi a^{4}\left(\mathcal{Q}\left(1+(-1)^{k+1} / k \pi\right)(1 / n \pi)\right)}{\left(1+2(n / m)^{2} r^{2}+(n / m)^{4} r^{4}\right)}\right) \Re,
\end{aligned}
$$

where $\mathfrak{R}$ denotes $\sin (m \pi x / a) \sin (n \pi y / b) \sin (k \pi z / c), \mathbb{Q}$ denotes $\left(n-\left(n^{2} / m\right) r^{3} / v^{2}\right)\left(1 / m^{3} \pi^{3}\right)$.

$$
\begin{aligned}
& \sum_{k=1}^{\infty} \sum_{n=1}^{\infty} \sum_{m=1}^{\infty}\left(\chi_{5}(m, n, k) \mathfrak{s}\right) \\
& =\sum_{k=1}^{\infty} \sum_{n=1}^{\infty} \sum_{m=1}^{\infty}\left(\frac{n}{m}\right) \frac{(\mathcal{T})}{\left(1+2(n / m)^{2} r^{2}+(n / m)^{4} r^{4}\right)} \mathfrak{s},
\end{aligned}
$$

where $\mathfrak{s}$ denotes $\sin (m \pi x / a) \sin (n \pi y / b) \sin (k \pi z / c), \quad \mathcal{T}$ denotes $\left(1+(-1)^{k+1} / k \pi\right)\left(a^{4} / m^{3} \pi^{3}\right)(1 / n \pi)$, in conjunction 
with the following Fourier closed form representations, namely,

$$
\begin{gathered}
\bar{q}=\frac{1}{\pi} \sum_{\xi=1}^{\infty} \frac{(-1)^{\xi+1}}{\xi} \sin \xi \pi \bar{q}, \quad \forall 0<\bar{q}<1, \\
\sum_{\xi=1}^{\infty} \frac{\sin \xi \pi \bar{q}}{\xi^{3}}=\frac{\pi^{2} \bar{q}}{6}-\frac{\pi \bar{q}^{2}}{4}+\frac{\bar{q}^{3}}{12}, \\
\forall 0<\bar{q}<2, \quad \forall(\bar{x}, \bar{y}, \bar{z}) \in \bar{q} .
\end{gathered}
$$

In view of the foregoing, (17) can now be written as

$$
\begin{aligned}
& \tilde{P}(s, \bar{x}, \bar{y}, \bar{z}) \\
& =c_{0}^{2}\left(\left(1-M^{2}\right) \bar{\Lambda}_{1}(\bar{x}, \bar{y}, \bar{z}) \mu \rho_{0}\left(1+\left(\psi_{1} / 2\right)\right)\right. \\
& +\bar{\Lambda}_{2}(\bar{x}, \bar{y}, \bar{z}) \mu \rho_{0}\left(1+\left(\psi_{2} / 2\right)\right)+\bar{\Lambda}_{3}(\bar{x}, \bar{y}, \bar{z}) \mu \rho_{\mathrm{av}} \Psi \\
& +\mathrm{c}\left(\bar{\Lambda}_{4}(\bar{x}, \bar{y}, \bar{z})\left(\frac{6 F_{01}}{E b H_{3}^{3}(s-i \omega)}-\frac{6 \mu \rho_{0}\left(1+\left(\psi_{2} / 2\right)\right)}{s E H_{z}^{2}}\right)\right. \\
& \left.\quad \times \bar{\Lambda}_{5}(\bar{x}, \bar{y}, \bar{z}) \mu r^{-1} b H_{z} \rho_{0}\left(\psi_{1}+r^{-1} \psi_{2}\right)\right) \\
& \left.\quad / \rho^{2}\left(s^{2}+\omega_{0}^{2}\right)\right) /(1+2 M)\left(s^{2}+\Omega^{2}\right) \\
& \times\left(1+2(n / m)^{2} r^{2}+(n / m)^{4} r^{4}\right) \omega_{0}^{-2}
\end{aligned}
$$

where the following have been defined, namely,

$$
\begin{aligned}
& \bar{\Lambda}_{1}(\bar{x}, \bar{y}, \bar{z})=\left(\frac{n}{m}\right) \frac{\left(1-v^{3}\right)}{a^{5} H_{z}} \sqrt{\frac{\rho}{E}}\left(32 \bar{x}-84 \bar{x}^{2}+10 \bar{x}^{3}\right) \overline{y z} ; \\
& \bar{\Lambda}_{2}(\bar{x}, \bar{y}, \bar{z})=a^{3} \sqrt{\frac{E}{\rho}}\left(\frac{n}{m}\right) \frac{3}{2}\left(\bar{x}^{2}-\bar{x}\right) \overline{y z} \\
& \bar{\Lambda}_{3}(\bar{x}, \bar{y}, \bar{z})=\frac{1}{c^{2}} \sqrt{\frac{E}{\rho}}\left(\frac{k}{m}\right)^{2} \frac{9}{4}\left(\bar{x}^{2}-\bar{x}\right) \overline{y z} ; \\
& \bar{\Lambda}_{4}(\bar{x}, \bar{y}, \bar{z})=\frac{c \pi a^{3}}{\rho^{2} b E H_{z}^{2}}\left(n-\left(\frac{n^{2}}{m}\right) \frac{r^{3}}{v^{2}}\right)\left(-2 \bar{x}+3 \bar{x}^{2}\right. \\
& \left.-\frac{2}{3} \bar{x}^{3}\right) \overline{y z} \\
& \bar{\Lambda}_{5}(\bar{x}, \bar{y}, \bar{z})=a^{3} \frac{H_{z}}{\rho}\left(\frac{n}{m}\right)\left(-2 \bar{x}+3 \bar{x}^{2}-\frac{2}{3} \bar{x}^{3}\right) \overline{y z},
\end{aligned}
$$

while

$$
\begin{gathered}
\Omega^{2}=\left(\frac{c_{0}^{2}}{1+2 M}\right)\left(\left(1-M^{2}\right) \frac{m^{2} \pi^{2}}{a^{2}}+\frac{n^{2} \pi^{2}}{b^{2}}+\frac{k^{2} \pi^{2}}{c^{2}}\right), \\
\omega_{0}^{2}=\frac{D}{\rho H_{z}}\left(\frac{m^{4} \pi^{4}}{a^{4}}+\frac{n^{4} \pi^{4}}{b^{4}}+\frac{k^{4} \pi^{4}}{c^{4}}\right)
\end{gathered}
$$

are the natural frequencies of the vibroacoustic enclosures. For the limit case as Mach number, $M \rightarrow 0$, the well known result in Laudau and Liftsitz [16] viz $\Omega^{2}=c_{0}^{2}\left(m^{2} \pi^{2} / a^{2}+\right.$ $\left.n^{2} \pi^{2} / b^{2}+k^{2} \pi^{2} / c^{2}\right)$, is correctly recovered. By employing the Laplace inversion, the closed form solution for the vibroacoustic pressure wave can be computed as

$$
\begin{aligned}
\bar{P}(\bar{x}, \bar{y}, \bar{z}, \tau) \\
=\Pi(m, n, r, M)\left(F _ { 1 } ( \tau ) \left(\left(1-M^{2}\right) \bar{\Lambda}_{1}(\bar{x}, \bar{y}, \bar{z}) \mu \bar{p}_{o} \mathfrak{r}\right.\right. \\
\left.+\bar{\Lambda}_{2}(\bar{x}, \bar{y}, \bar{z}) \mu \bar{p}_{o}\left(1+\frac{\psi_{2}}{2}\right)+\bar{\Lambda}_{3}(\bar{x}, \bar{y}, \bar{z}) \mu \bar{p}_{0} \Psi\right) \\
+\left(\bar{\Lambda}(\bar{x}, \bar{y}, \bar{z})\left(6 F_{2}(\tau)-6 \mu \bar{p}_{0} F_{3}(\tau)\left(1+\frac{\psi_{2}}{2}\right)\right)\right. \\
\left.+\bar{\Lambda}_{5}(\bar{x}, \bar{y}, \bar{z}) F_{4}(\tau) r^{-1} \mu \bar{p}_{0}\left(\psi_{1}+r^{-1} \psi_{2}\right)\right),
\end{aligned}
$$

where $\mathfrak{r}$ denotes $\left(1+\left(\psi_{1} / 2\right)\right)$,

$$
\begin{aligned}
& \forall F_{1}(\tau)=\kappa \frac{\sin 2 \pi \eta \tau}{\eta} ; \quad \bar{P}(\bar{x}, \bar{y}, \bar{z}, \tau)=\frac{P(\bar{x}, \bar{y}, \bar{z}, \tau)}{\left(F / b H_{z}\right)}, \\
& \bar{p}_{0}=\frac{p_{0}}{\left(F / b H_{z}\right)}, \quad t=\frac{2 \pi \tau}{\omega_{0}}, \\
& F_{2}(\tau)=\kappa^{2}\left(\frac{e^{i 2 \pi \eta \tau}}{\left(1-\eta_{1}^{2}\right)\left(\eta^{2}-\eta_{1}^{2}\right)}\right. \\
& +\left(\frac{\cos 2 \pi \tau+i \eta_{1} \sin 2 \pi \tau}{\left(1-\eta^{2}\right)\left(1-\eta_{1}^{2}\right)}\right) \\
& \left.-\frac{\cos 2 \eta \pi \tau}{\eta\left(1-\eta^{2}\right)\left(\eta-\eta_{1}\right)}\right) \\
& F_{3}(\tau)=\kappa^{2}\left(\frac{1}{\eta^{2}}-\frac{e^{i 2 \pi \tau}}{2\left(1-\eta^{2}\right)}-\frac{e^{-i 2 \pi \tau}}{2\left(1-\eta^{2}\right)}\right. \\
& \left.-\frac{e^{i 2 \pi \tau}}{2 \eta^{2}\left(1-\eta^{2}\right)}-\frac{e^{-i 2 \pi \tau}}{2 \eta^{2}\left(1-\eta^{2}\right)}\right), \\
& F_{4}(\tau)=\kappa\left(\frac{\sin 2 \pi \eta+\eta \sin 2 \pi \tau}{2 \eta^{3}\left(1-\eta^{2}\right)}\right) \text {, } \\
& \kappa=\frac{c_{m}}{\omega_{0}}, \quad \eta=\frac{\Omega}{\omega_{0}}, \quad \eta_{1}=\frac{\omega}{\omega_{0}}, \\
& \Pi(m, n, r, M)=(1+2 M)\left(1+2\left(\frac{n}{m}\right)^{2} r^{2}\right. \\
& \left.+\left(\frac{n}{m}\right)^{4} r^{4}\right)
\end{aligned}
$$

\section{Mathematical Analysis of Sound Intensity as Influenced by Interfacial Pressure Distribution}

In acoustics, sound intensity $\vec{I}$, is a vector quantity and can be defined as the time average of the net flow of sound 


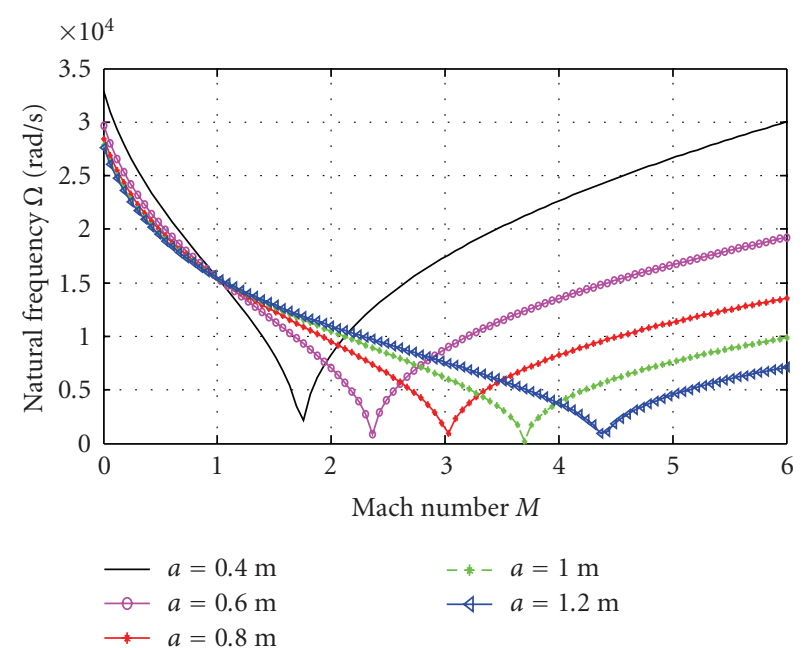

FIGURE 6: Acoustic natural frequency profile for the case $m=7 ; n=$ $7 ; k=7 ; b=0.3 ; c=0.8$.

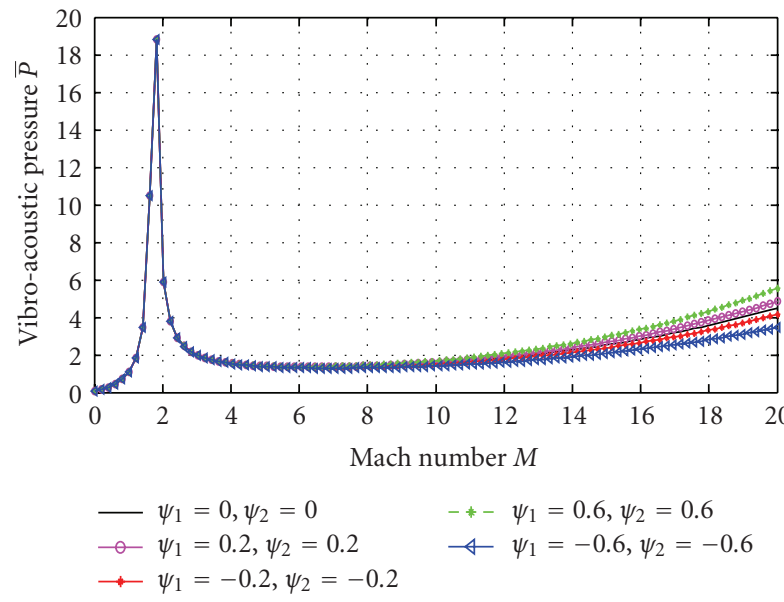

Figure 7: Vibroacoustic pressure profile for the case $m=1 ; n=$ $1 ; k=1 ; b=0.3 \mathrm{~m} ; c=0.8 \mathrm{~m} ; \bar{x}=0.1270 ; \bar{y}=0.1905 ; \bar{z}=$ $-0.3112 ; \omega=10 \mathrm{~Hz}$.

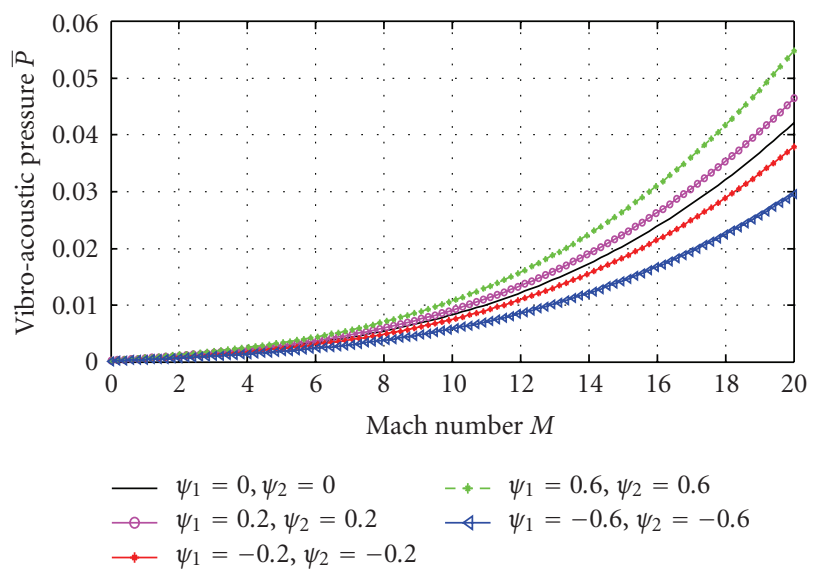

Figure 8: Vibroacoustic pressure profile for the case $m=1 ; n=$ $1 ; k=1 ; b=0.3 \mathrm{~m} ; c=0.8 \mathrm{~m} ; \bar{x}=0.1270 ; \bar{y}=0.1905 ; \bar{z}=$ $-0.3112 ; \omega=1 \mathrm{kHz}$.

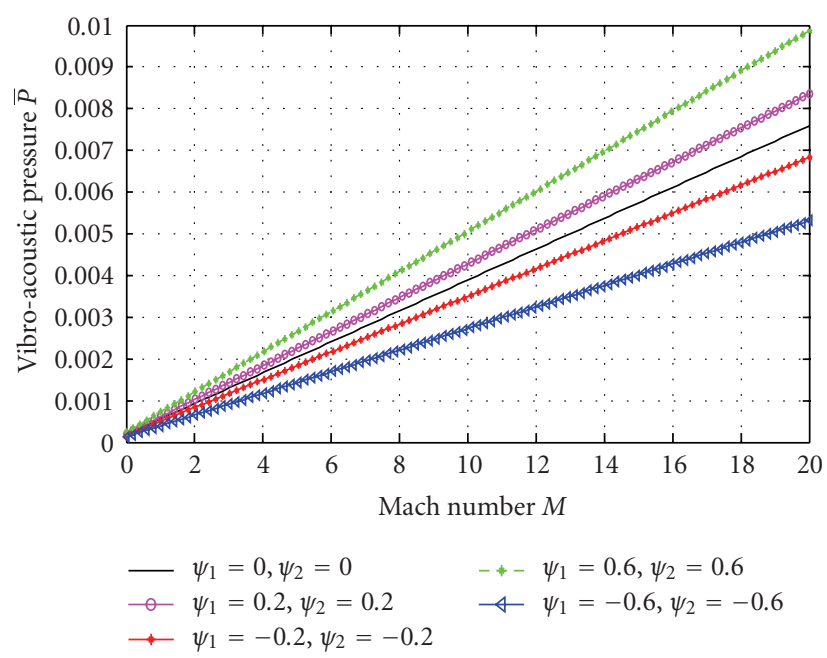

FIGURE 9: Vibroacoustic pressure profile for the case $m=1 ; n=$ $1 ; k=2 ; b=0.3 \mathrm{~m} ; c=0.8 \mathrm{~m} ; \bar{x}=0.1270 ; \bar{y}=0.1905 ; \bar{z}=$ $-0.3112 ; \omega=1 \mathrm{MHz}$.

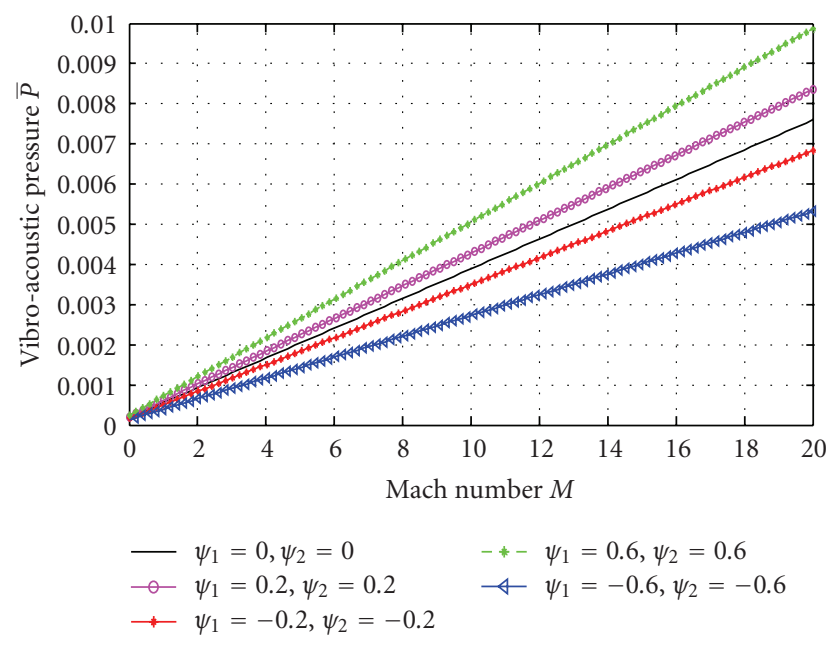

FIGURE 10: Vibroacoustic pressure profile for the case $m=1 ; n=$ $1 ; k=1 ; b=0.3 \mathrm{~m} ; c=0.8 \mathrm{~m} ; \bar{x}=0.1270 ; \bar{y}=0.1905 ; \bar{z}=$ $-0.3112 ; \omega=1 \mathrm{GHz}$.

energy per unit area in a direction normal to the area. By combining the fundamental equations governing sound field, mass continuity, the relation between sound pressure and density change in conjunction with Euler's equation of motion, leads to the equation relating sound intensity with the net flow of energy, viz,

$$
\vec{\nabla} \cdot \vec{I}(t)+\frac{\partial\left(E_{k \cdot e}+E_{p \cdot e}\right)}{\partial t}=0,
$$

where $\left(E_{k \cdot e}+E_{p \cdot e}\right)$ is total acoustic energy.

In this paper, we shall limit analysis to intensity variation along the spatial variable $x$ and for this purpose; the forgoing equation is modified to the following form, namely,

$$
\|\vec{I}(t)\|=\int_{0}^{x}\left(\frac{\partial P}{\partial t} A_{c} \zeta+P A_{c} c_{m}\right) d \zeta,
$$




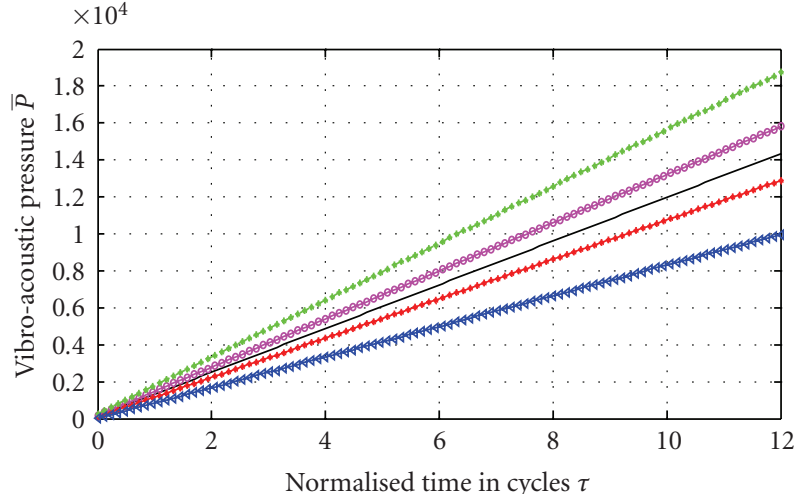

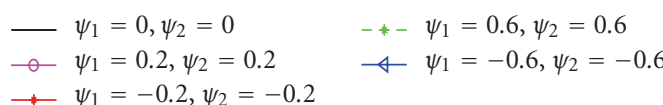

FIGURE 11: Vibroacoustic pressure profile for the case $m=1 ; n=$ $1 ; k=1 ; b=0.3 \mathrm{~m} ; c=0.8 \mathrm{~m} ; \bar{x}=0.1270 ; \bar{y}=0.1905 ; \bar{z}=$ $-0.3112 ; \omega=10 \mathrm{~Hz}$.

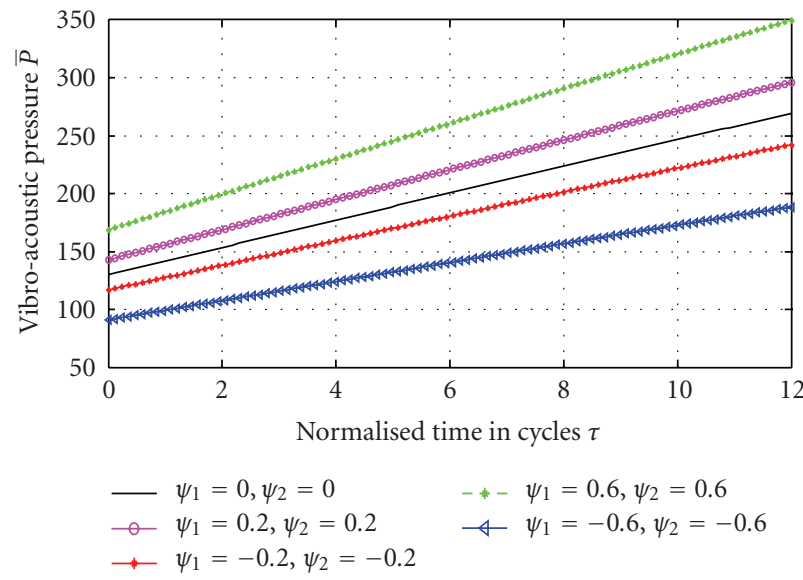

Figure 12: Vibroacoustic pressure profile for the case $m=1 ; n=$ $1 ; k=1 ; b=0.3 \mathrm{~m} ; c=0.8 \mathrm{~m} ; \bar{x}=0.1270 ; \bar{y}=0.1905 ; \bar{z}=$ $-0.3112 ; \omega=1 \mathrm{kHz}$.

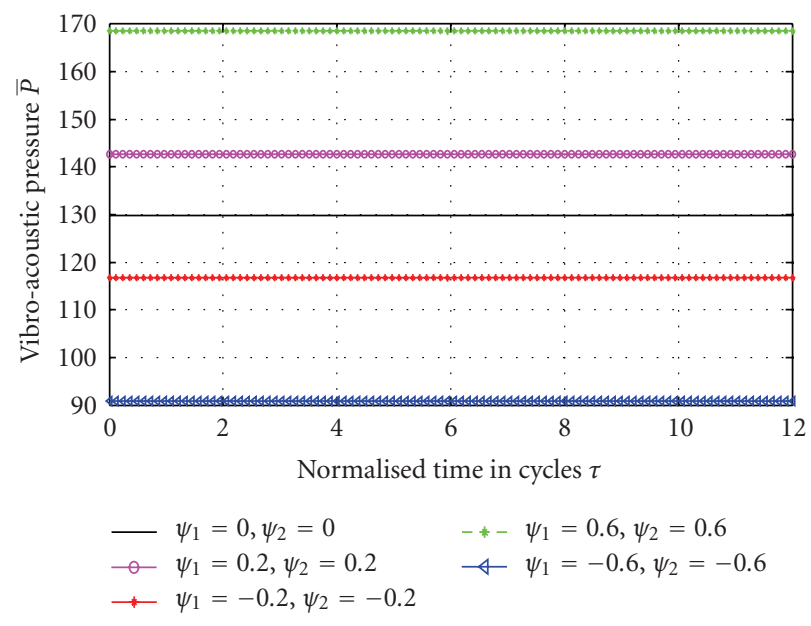

FIGURE 13: Vibroacoustic pressure profile for the case $m=1 ; n=$ $1 ; k=1 ; b=0.3 \mathrm{~m} ; c=0.8 \mathrm{~m} ; \bar{x}=0.1270 ; \bar{y}=0.1905 ; \bar{z}=$ $-0.3112 ; \omega=1 \mathrm{MHz}$. where $P$ is the vibroacoustic pressure; $A_{c}$ is the cross sectional area of the 3-D enclosure; $c_{m}$ is the velocity of sound through the $3-\mathrm{D}$ enclosure; $\zeta$ is a dummy variable of integration. Equation (31) can be normalized into to the form

$$
\begin{aligned}
\|\vec{I}(\tau)\|= & \int_{0}^{\bar{x}}\left(\frac{\partial \bar{P}}{\partial \tau} \bar{\zeta}+\bar{P} M\right) \mathrm{d} \zeta \\
& \forall \vec{I}(\tau)=\frac{\vec{I}(t) b H_{z}}{A_{c} F L c_{0}}, \quad \frac{L}{t_{0} c_{0}}=1, \quad t=t_{0} \tau \\
& \bar{P}=\bar{P}(\bar{\zeta}, \bar{y}, \bar{z}, \tau)
\end{aligned}
$$

in conjunction with the ongoing mathematical representations, viz,

$$
\begin{aligned}
& \gamma_{1}(\bar{x}, \bar{y}, \bar{z})=\left(\frac{n}{m}\right) \frac{\left(1-v^{3}\right)}{a^{5} H_{z}} \sqrt{\frac{\rho}{E}}\left(16 \bar{x}^{2}-28 \bar{x}^{3}+\frac{5}{2} \bar{x}^{4}\right) \overline{y z}, \\
& \gamma_{2}(\bar{x}, \bar{y}, \bar{z})=a^{3} \sqrt{\frac{E}{\rho}}\left(\frac{n}{m}\right)\left(\frac{3 \bar{x}^{4}}{8}-\frac{\bar{x}^{3}}{2}\right) \overline{y z}, \\
& \gamma_{3}(\bar{x}, \bar{y}, \bar{z})=\frac{1}{c^{2}} \sqrt{\frac{E}{\rho}}\left(\frac{k}{m}\right)^{2}\left(\frac{9 \bar{x}^{3}}{16}-\frac{3}{4} \bar{x}^{2}\right) \overline{y z} \\
& \gamma_{4}(\bar{x}, \bar{y}, \bar{z}) \\
& =\frac{c \pi a^{3}}{\rho^{2} b E H_{z}^{2}}\left(n-\left(\frac{n^{2}}{m}\right) \frac{r^{3}}{v^{2}}\right)\left(-\frac{2 \bar{x}^{2}}{3}+\frac{3 \bar{x}^{3}}{4}-\frac{2 \bar{x}^{4}}{15}\right) \overline{y z}, \\
& \gamma_{5}(\bar{x}, \bar{y}, \bar{z})=a^{3} \frac{H_{z}}{\rho}\left(\frac{n}{m}\right)\left(-\frac{2 \bar{x}^{2}}{3}+\frac{3 \bar{x}^{3}}{4}-\frac{2 \bar{x}^{4}}{15}\right) \overline{y z}, \\
& \gamma_{6}(\bar{x}, \bar{y}, \bar{z})=\left(\frac{n}{m}\right) \frac{\left(1-v^{3}\right)}{a^{5} H_{z}} \sqrt{\frac{\rho}{E}}\left(16 \bar{x}^{2}-28 \bar{x}^{3}+\frac{5}{2} \bar{x}^{4}\right) \overline{y z}, \\
& \gamma_{7}(\bar{x}, \bar{y}, \bar{z})=a^{3} \sqrt{\frac{E}{\rho}}\left(\frac{n}{m}\right)\left(\frac{\bar{x}^{3}}{2}-\frac{3 \bar{x}^{2}}{4}\right) \overline{y z}, \\
& \gamma_{8}(\bar{x}, \bar{y}, \bar{z})=\frac{1}{c^{2}} \sqrt{\frac{E}{\rho}}\left(\frac{k}{m}\right)^{2}\left(\frac{3 \bar{x}^{3}}{4}-\frac{9}{8} \bar{x}^{2}\right) \overline{y z} \\
& \gamma_{9}(\bar{x}, \bar{y}, \bar{z})=\frac{c \pi a^{3}}{\rho^{2} b E H_{z}^{2}}\left(n-\left(\frac{n^{2}}{m}\right) \frac{r^{3}}{v^{2}}\right)\left(-\bar{x}^{2}+\bar{x}^{3}-\frac{1}{6} \bar{x}^{4}\right) \overline{y z}, \\
& \gamma_{10}(\bar{x}, \bar{y}, \bar{z})=a^{3} \frac{H_{z}}{\rho}\left(\frac{n}{m}\right)\left(-\bar{x}^{2}+\bar{x}^{3}-\frac{1}{6} \bar{x}^{4}\right) \overline{y z}, \\
& \mathrm{H}_{1}(\tau)=2 \kappa \pi \cos 2 \pi \eta \tau, \quad t=\frac{2 \pi \tau}{\omega_{0}}, \\
& \mathrm{H}_{2}(\tau)=\kappa^{2}\left(\frac{2 \pi \eta i e^{i 2 \pi \eta \tau}}{\left(1-\eta_{1}^{2}\right)\left(\eta^{2}-\eta_{1}^{2}\right)}\right. \\
& +\left(\frac{-2 \pi \sin 2 \pi \tau+i 2 \pi \eta_{1} \cos 2 \pi \tau}{\left(1-\eta^{2}\right)\left(1-\eta_{1}^{2}\right)}\right)
\end{aligned}
$$




$$
\begin{gathered}
\left.+\frac{2 \eta \pi \sin 2 \eta \pi \tau}{\eta\left(1-\eta^{2}\right)\left(\eta-\eta_{1}\right)}\right), \\
\mathrm{H}_{3}(\tau)=\kappa^{2}\left(-\frac{2 \pi i e^{i 2 \pi \tau}}{2\left(1-\eta^{2}\right)}+\frac{2 \pi i e^{-i 2 \pi \tau}}{2\left(1-\eta^{2}\right)}\right. \\
\left.-\frac{2 \pi i e^{i 2 \pi \tau}}{2 \eta^{2}\left(1-\eta^{2}\right)}+\frac{2 \pi i e^{-i 2 \pi \tau}}{2 \eta^{2}\left(1-\eta^{2}\right)}\right), \\
\mathrm{H}_{4}(\tau)=\kappa\left(\frac{-\pi \cos 2 \pi \eta+\pi \eta \sin 2 \pi \tau}{\eta^{2}\left(1-\eta^{2}\right)}\right) .
\end{gathered}
$$

In view of the above relations, the magnitude of the sound intensity can be evaluated from (35) as

$$
\begin{aligned}
& \|\vec{I}(\tau)\| \\
& =\Pi(m, n, r, M) \\
& \times\left(\mathrm { H } _ { 1 } ( \tau ) \left(\left(1-M^{2}\right) \gamma_{1}(\bar{x}, \bar{y}, \bar{z}) \mu \bar{p}_{o}\left(1+\frac{\psi_{1}}{2}\right)\right.\right. \\
& +\gamma_{2}(\bar{x}, \bar{y}, \bar{z}) \mu \bar{p}_{o}\left(1+\frac{\psi_{2}}{2}\right) \\
& \left.+\gamma_{3}(\bar{x}, \bar{y}, \bar{z}) \mu \bar{p}_{0} \Psi\right) \\
& +\left(\gamma_{4}(\bar{x}, \bar{y}, \bar{z})\left(6 \mathrm{H}_{2}(\tau)-6 \mu \bar{p}_{0} \mathrm{H}_{3}(\tau)\left(1+\frac{\psi_{2}}{2}\right)\right)\right. \\
& \left.+\gamma_{5}(\bar{x}, \bar{y}, \bar{z}) \mathrm{H}_{4}(\tau) r^{-1} \mu \bar{p}_{0}\left(\psi_{1}+r^{-1} \psi_{2}\right)\right) \\
& +M\left(F _ { 1 } ( \tau ) \left(\left(1-M^{2}\right) \gamma_{6}(\bar{x}, \bar{y}, \bar{z}) \mu \bar{p}_{o}\left(1+\frac{\psi_{1}}{2}\right)\right.\right. \\
& +\gamma_{7}(\bar{x}, \bar{y}, \bar{z}) \mu \bar{p}_{o}\left(1+\frac{\psi_{2}}{2}\right) \\
& \left.+\gamma_{8}(\bar{x}, \bar{y}, \bar{z}) \mu \bar{p}_{0} \Psi\right) \\
& +\left(\gamma_{8}(\bar{x}, \bar{y}, \bar{z})\left(6 F_{2}(\tau)-6 \mu \bar{p}_{0} F_{3}(\tau)\left(1+\frac{\psi_{2}}{2}\right)\right)\right. \\
& \left.\left.\left.+\gamma_{10}(\bar{x}, \bar{y}, \bar{z}) F_{4}(\tau) r^{-1} \mu \bar{p}_{0}\left(\psi_{1}+r^{-1} \psi_{2}\right)\right)\right)\right) .
\end{aligned}
$$

\section{Analysis of Results}

In this paper, vibration and noise propagation control emanating from complex engineering systems such as industrial power plants, aircraft engines, space propulsive devices and machine enclosures is investigated. The acoustic-structure configuration of interest is the one in which an acoustic disturbance is prompted by one of the vibrating boundaries of the enclosure such as in aircraft cabin noise transmission and systems for outer space exploration. The modeling techniques employed for this study derives from recent advances made in the mechanics of sandwich structures, with non-uniform interfacial pressure distribution. The acousticstructure dynamic interaction problem is simplified by

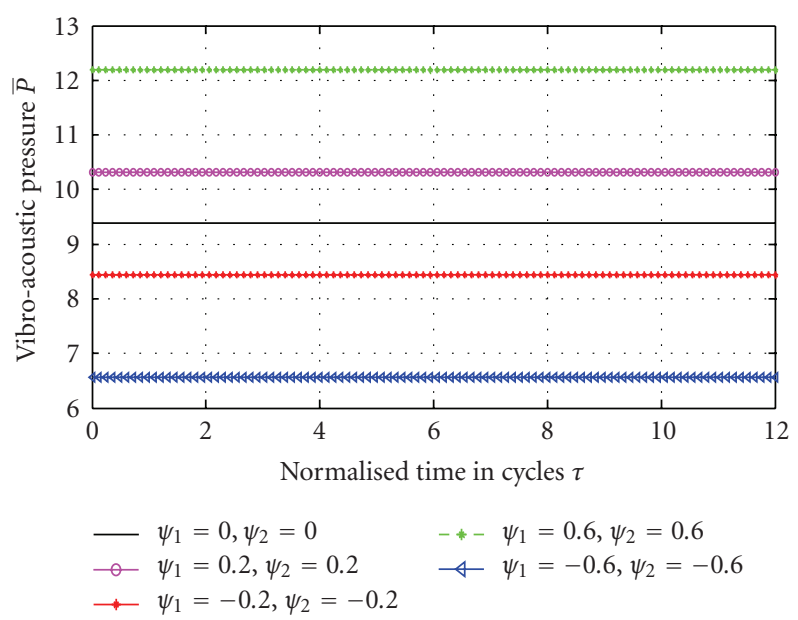

FIGURE 14: Vibroacoustic pressure profile for the case $m=2 ; n=$ $1 ; k=1 ; b=0.3 \mathrm{~m} ; c=0.8 \mathrm{~m} ; \bar{x}=0.1270 ; \bar{y}=0.1905 ; \bar{z}=$ $-0.3112 ; \omega=1 \mathrm{GHz}$.

assuming zero initial conditions prior to the excitation of the upper boundary surface. In the formulated problem, the upper layer of the sandwich elastic plates is subjected to harmonic excitation force as illustrated in Figure 1. To demonstrate the practical significant of our model problem, we have utilized some characteristic values listed in [10] as shown in the Canadian Society for Mechanical Engineers (CSME), Transaction of Applied Mechanics for simulation. Figures 2, 3, 4, 5, and 6 illustrate the characteristic acoustic natural frequencies as couched in (29). In fact, our expression allows for the incorporation of Mach number and for the case where the Mach number is approaching zero, Landau and Lifshitz [16] characteristic frequencies equation is recovered as a special case. As can be seen, the ordering of the family of curves clearly displayed the existence of subsonic, sonic, supersonic and hypersonic zones.

In each zone, the general pattern of the frequency profiles showed that the results are dependent on the geometry of the 3-D enclosure and ambient sound velocity. With respect to the effect of modal parameters, Figure 2, showed the profiles for the principal modes as the axial length increases from 0.4 to $1.2 \mathrm{~m}$ whilst $b$ and $c$ are restricted to the values listed in Table 1 .

In the subsonic zone, the natural frequency profiles are inversely proportional to the axial length of the enclosure and decrease monotonically to a constant value irrespective of the axial length as we approach sonic flow. However, in the supersonic zone, the natural frequencies are further attenuated in reversed order to their respective critical values before increasing monotonically in the hypersonic zone.

With respect to the effect of higher modal parameters on the natural frequency profiles, as demonstrated in Figures 36 , we observed similar pattern but with higher magnitude.

With respect to the profiles of vibroacoustic pressure, Figures $7,8,9,10,11,12,13,14,15$, and 16 displayed the various pictures for low and very high frequency excitations. In general the pressure wave profiles are strongly influenced 
TABLE 1: Parameters for simulation of results.

\begin{tabular}{lcc}
\hline Definition & Symbol & Value \\
\hline Applied force amplitude & $F_{01}$ & $1 \mathrm{MN}$ \\
Poisson ratio & $v$ & 0.33 \\
Coefficient of friction & $\mu$ & 0.14 \\
Interfacial pressure & $\mathrm{P}$ & $1 \times 10^{9} \mathrm{Nm}^{-2}$ \\
3-D: - Length & $a$ & $0.4 \mathrm{~m}-1.2 \mathrm{~m}$ \\
$\quad$-Width & $b$ & $0.3 \mathrm{~m}$ \\
$\quad$-Thickness & $c$ & $0.8 \mathrm{~m}$ \\
Thickness of laminated boundary surface on $z$-axis & $H_{z}$ & $0.005 \mathrm{~m}$ \\
Excitation frequency & $\omega$ & $1 \mathrm{HZ}^{-1 \mathrm{mHz}^{-2}}$ \\
Modulus of rigidity of materials & $E$ & $7.1 \times 10^{10} \mathrm{Nm}^{-2}$ \\
Velocity of Sound through the 3-D enclosure & $C_{\mathrm{o}}$ & $343 \mathrm{~ms}^{-1}$ \\
Density of material & $\rho$ & $2810 \mathrm{~kg} \mathrm{~m}^{-3}$ \\
Density of air & $\rho_{\text {air }}$ & $1.13 \mathrm{~kg} \mathrm{~m}^{-3}$ \\
\hline
\end{tabular}

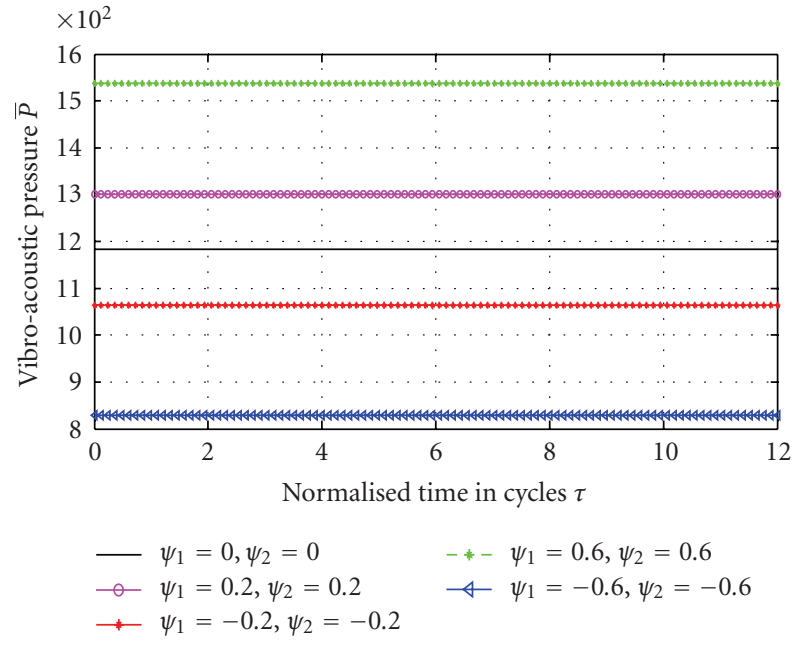

Figure 15: Vibroacoustic pressure profile for the case $m=1 ; n=$ $2 ; k=1 ; b=0.3 \mathrm{~m} ; c=0.8 \mathrm{~m} ; \bar{x}=0.1270 ; \bar{y}=0.1905 ; \bar{z}=$ $-0.3112 ; \omega=1 \mathrm{GHz}$.

by the values of the pressure gradients and the frequency of the vibrating boundary surface. Interestingly, several profiles can be simulated by playing with the values of $\psi_{1}$ and $\psi_{2}$ irrespective of the frequency of excitation, whereas the choice of any suitable combination can be arranged by progressively varying the tightening torques along the laminated surfaces. Such pressure gradients selection can have significant effect on the vibroacoustic pressure levels.

The pictures in Figures 7-10 illustrate the acoustic pressure profiles at the end of one cycle of vibration.

With respect to the effect of low excitation frequency through the boundary surface, the interfacial pressure gradients do not seem to have any strong effect on the acoustic pressure profiles. In fact we note in the lower range of Mach number, that the acoustic pressure waves rise initially before dropping at Mach 2 and increased marginally. However, as we move beyond Mach 16, the effects of the pressure gradients are noticeable.

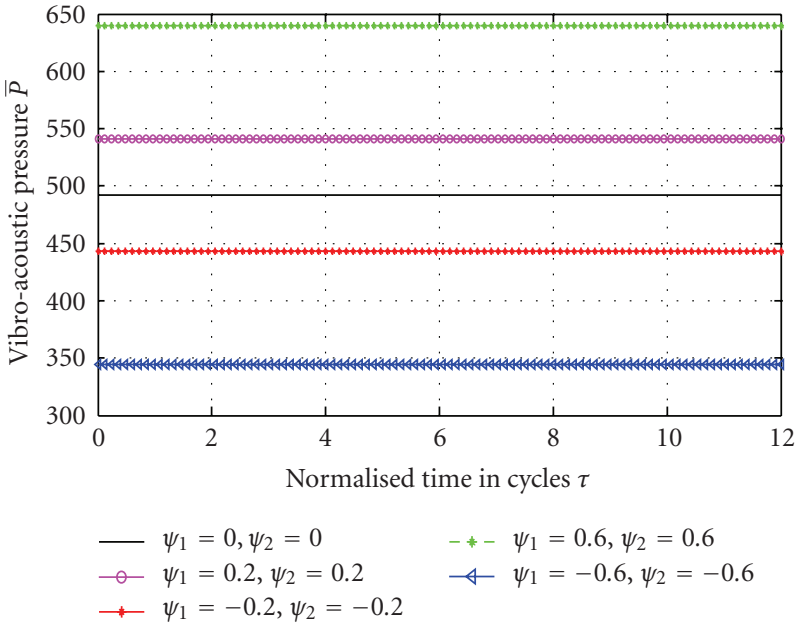

FIGURE 16: Vibroacoustic pressure profile for the case $m=1 ; n=$ $1 ; k=2 ; b=0.3 \mathrm{~m} ; c=0.8 \mathrm{~m} ; \bar{x}=0.1270 ; \bar{y}=0.1905 ; \bar{z}=$ $-0.3112 ; \omega=1 \mathrm{GHz}$.

On the other hand, Figures 8-11 displayed the pattern of the acoustic pressure waves from low to high excitation frequency in the vibrating boundary surface. We observed that the magnitudes of the pressure waves are significantly reduced with higher excitation frequency. Nonetheless, Figures 11-16 illustrate the vibroacoustic pressure profile for the principal modes at Mach 3, as a function of the normalized cycles of vibration.

As can be observed, the pressure profiles are increasing with time. In particular, we note that pressure profile magnitude with lower boundary excitation frequency are significantly higher compare with the effect of higher boundary excitation frequency. On the other hand, Figure 13, showed the acoustic pressure profiles for an excitation frequency of $1 \mathrm{MHz}$. In respective of the pressure gradients combination, the profiles are monostatically stable. As the boundary excitation frequency increases to $1 \mathrm{GHz}$, the pressure profiles are significantly lower in the fundamental mode of vibration. 
However, this may not be the case as we vary the values of the modal parameters by comparison of Figures 14-16. This suggests that in practice, dynamic stability of hypersonic aircrafts or jet airplanes can be further enhanced by replacing their noise transmission systems with laminated enclosures.

\section{Summary and Conclusion}

In this paper, explicit closed form solutions for the vibroacoustic characteristic frequencies, pressure waves and sound intensity or transmission quality through a 3-D enclosure with a vibrating laminated boundary surface is investigated. The vibroacoustic properties are shown to be dependent on, interfacial pressure gradients and boundary surfaces excitation frequencies. The results presented in this study can be positively exploited for the design of modern airplanes, aeroelastic structures and propulsive devices for the launching of space systems.

\section{Appendix}

For Case $\Omega_{1}$,

$$
\begin{gathered}
D\left(\frac{\partial^{4} W_{1}}{\partial x^{4}}+2 \frac{\partial^{4} W_{1}}{\partial x^{2} \partial y^{2}}+\frac{\partial^{4} W_{1}}{\partial y^{4}}\right)+\rho_{1} h \frac{\partial^{2} W_{1}}{\partial t^{2}} \\
-\left(\frac{\mu H_{z}}{2} \frac{\partial p(x, 0)}{\partial x}+\frac{\mu H_{z}}{2} \frac{\partial p(0, y)}{\partial y}\right)=0 .
\end{gathered}
$$

For Case $\Omega_{2}$,

$$
\frac{\partial^{4} W_{2}}{\partial x^{4}}+\beta_{2} \frac{\partial^{2} W_{2}}{\partial t^{2}}=\alpha_{2} \frac{\partial P(x, 0)}{\partial x} .
$$

For Case $\Omega_{3}$ (mirror reflection of $\Omega_{1}$ ),

$$
\begin{gathered}
D\left(\frac{\partial^{4} W_{3}}{\partial x^{4}}+2 \frac{\partial^{4} W_{3}}{\partial x^{2} \partial y^{2}}+\frac{\partial^{4} W_{3}}{\partial y^{4}}\right)+\rho_{1} h \frac{\partial^{2} W_{3}}{\partial t^{2}} \\
-\left(\frac{\mu H_{z}}{2} \frac{\partial p(x, 0)}{\partial x}+\frac{\mu H_{z}}{2} \frac{\partial p(0, y)}{\partial y}\right)=0 .
\end{gathered}
$$

For Case $\Omega_{4}$ (mirror reflection of $\Omega_{2}$ ),

$$
\begin{gathered}
\frac{\partial^{4} W_{4}}{\partial x^{4}}+\beta_{4} \frac{\partial^{2} W_{4}}{\partial t^{2}}=\alpha_{4} \frac{\partial P(x, 0)}{\partial x}, \\
\forall \alpha_{1}=\alpha_{2}=\alpha_{3}=\alpha_{4}=\frac{6 \mu}{E h^{2}}, \quad \beta_{1}=\beta_{2}=\beta_{3}=\beta_{4}=\frac{\rho b h}{E I} .
\end{gathered}
$$

Now for the 3-D cantilever enclosures under investigation, the following boundary conditions hold for each of the vibrating membranes, viz,
For Case $\Omega_{1}$,

(i) $W_{1}(0,0, t)=W_{1}(0, b, t)=0, \quad \forall z=0$,

(ii) $\frac{\partial W_{1}(0,0, t)}{\partial x}=\frac{\partial W_{1}(0, b, t)}{\partial x}=0, \quad \forall z=0$,

(iii) $\frac{\partial W_{1}^{2}(a, 0, t)}{\partial x^{2}}=\frac{\partial W_{1}^{2}(a, b, t)}{\partial x^{2}}=0, \quad \forall z=0$,

(iv) $W_{1}(a, 0, t)=W_{1}(a, b, t)=0, \quad \forall z=0$,

(v) $\frac{\partial W_{1}(0,0, t)}{\partial y}=\frac{\partial W_{1}(0, b, t)}{\partial y}=0, \quad \forall z=0$,

(vi) $\frac{\partial W_{1}^{2}(a, 0, t)}{\partial y^{2}}=\frac{\partial W_{1}^{2}(a, b, t)}{\partial y^{2}}=0, \quad \forall z=0$.

For Case $\Omega_{2}$,

(i) $\quad W_{2}(0,0, t)=\frac{\partial W_{2}(0,0, t)}{\partial x}=\frac{\partial W_{2}^{2}(a, 0, t)}{\partial x^{2}}=0$,

For Case $\Omega_{3}$,

(i) $W_{3}(0,0, t)=W_{3}(0, b, t)=0, \quad \forall z=0$,

(ii) $\frac{\partial W_{3}(0,0, t)}{\partial x}=\frac{\partial W_{3}(0, b, t)}{\partial x}=0, \quad \forall z=-h$,

(iii) $\frac{\partial W_{3}^{2}(a, 0, t)}{\partial x^{2}}=\frac{\partial W_{3}^{2}(a, b, t)}{\partial x^{2}}=0, \quad \forall z=-h$

(iv) $W_{3}(a, 0, t)=W_{3}(a, b, t)=0, \quad \forall z=-h$

(v) $\frac{\partial W_{3}(0,0, t)}{\partial y}=\frac{\partial W_{3}(0, b, t)}{\partial y}=0, \quad \forall z=-h$

(vi) $\frac{\partial W_{3}^{2}(a, 0, t)}{\partial y^{2}}=\frac{\partial W_{3}^{2}(a, b, t)}{\partial y^{2}}=0, \quad \forall z=-h$.

For Case $\Omega_{4}$,

$$
\begin{gathered}
W_{4}(0, b, t)=\frac{\partial W_{4}(0, b, t)}{\partial x}=\frac{\partial W_{4}^{2}(a, b, t)}{\partial x^{2}}=0, \\
\forall z=-h
\end{gathered}
$$

On the other hand the effects of the excitation forces at the free end of the 3-D cantilever enclosures can be captured via the following end conditions [11].

For case $\Omega_{1}$,

(i) $\int_{0}^{H_{y}} \tau_{(x z)_{1(1)}}(x, t)=\frac{F_{1}(t)}{2 b}$, at $x=a$, 


$$
\begin{aligned}
& \forall \tau_{(x z)_{1(1)}}\left(x, y, H_{z}\right) \\
& =\frac{E\left(z^{2}+z H_{z}\right)}{2\left(1-v^{2}\right)}\left(\frac{\partial^{3} W_{3}}{\partial x^{3}}+v \frac{\partial^{3} W_{3}}{\partial x \partial y^{2}}\right) \\
& \quad+\left(\frac{E\left(z^{2}+z H_{z}\right)(1-v)}{2\left(1-v^{2}\right)}\right) \frac{\partial^{3} W_{3}}{\partial x \partial y^{2}}+\frac{\mu p(x, 0)\left(z+H_{z}\right)}{H_{z}},
\end{aligned}
$$

(ii) $\int_{0}^{H_{y}} \tau_{(y z)_{1(1)}}(x, t)=\frac{T R_{1} F_{1}(t)}{2 b}$, at $x=a$,

$\forall T R<1$,

$\forall \tau_{(y z)_{1}}\left(x, y, H_{z}\right)$

$=\left(\frac{E\left(z^{2}+z H_{z}\right)}{2\left(1-v^{2}\right)}\left(\frac{\partial^{3} W_{3}}{\partial y^{3}}+v \frac{\partial^{3} W_{3}}{\partial x^{2} \partial y}\right)\right.$

$\left.+\left(\frac{E\left(z^{2}+z H_{z}\right)(1-v)}{2\left(1-v^{2}\right)}\right) \frac{\partial^{2} W_{3}}{\partial y \partial x^{2}}+\frac{\mu p(x, 0)\left(z+H_{z}\right)}{H_{z}}\right)$

For case $\Omega_{2}$,

$$
\begin{aligned}
& \int_{0}^{H_{y}} \tau_{(x y)_{2(1)}}(x, t)=\frac{-F_{2}(t)}{2 h}, \text { at } x=a, \\
& \forall \tau_{(x y)_{2(1)}}(x, t) \\
& =\frac{E}{2}\left(y^{2}-H_{y} y\right) \frac{\partial^{3} W_{2}}{\partial x^{3}}-\frac{\mu p\left(y-H_{y}\right)}{H_{y}} .
\end{aligned}
$$

For case $\Omega_{3}$,

(i) $\int_{-H_{y}}^{0} \tau_{(x z)_{3(1)}}(x, t)=\frac{F_{3}(t)}{2 b}$, at $x=a$,

$$
\forall \tau_{(x z)}\left(x, y,-H_{z}\right)
$$

$$
\begin{aligned}
= & \frac{E\left(z^{2}+z H_{z}\right)}{2\left(1-v^{2}\right)}\left(\frac{\partial^{3} W_{3}}{\partial x^{3}}+v \frac{\partial^{3} W_{3}}{\partial x \partial y^{2}}\right) \\
& +\left(\frac{E\left(z^{2}+z H_{z}\right)(1-v)}{2\left(1-v^{2}\right)}\right) \frac{\partial^{3} W_{3}}{\partial x \partial y^{2}}+\frac{\mu p(x, 0)\left(z+H_{z}\right)}{H_{z}}
\end{aligned}
$$

(ii) $\int_{-H_{y}}^{0} \tau_{(y z)_{3(1)}}(x, t)=\frac{T R_{3} F_{3}(t)}{2 b}, \quad$ at $x=a, \quad \forall T R<1$,

$$
\begin{aligned}
& \forall \tau_{(y z)}\left(x, y-H_{z}\right) \\
& =\frac{E\left(z^{2}+z H_{z}\right)}{2\left(1-v^{2}\right)}\left(\frac{\sigma^{3} W_{3}}{\sigma y^{3}}+v \frac{\sigma^{3} W_{3}}{\sigma x^{2} \sigma y}\right) \\
& \quad+\left(\frac{E\left(z^{2}+z H_{z}\right)(1-v)}{2\left(1-v^{2}\right)}\right) \frac{\sigma^{2} W_{3}}{\sigma y \sigma x^{2}}+\frac{\mu \rho(x, 0)\left(z+H_{z}\right)}{H_{z}} .
\end{aligned}
$$

For case $\Omega_{4}$,

$$
\begin{aligned}
& \int_{-H_{y}}^{0} \tau_{(x y)_{4(1)}}(x, t)=\frac{F_{4}(t)}{2 h}, \quad \text { at } x=0, \\
& \forall \tau_{(x y)_{4(1)}}(x, t) \\
& =\frac{E}{2}\left(y^{2}+H_{y} y\right) W_{4 x x x}(x, t)+\frac{\mu p\left(y+H_{y}\right)}{H_{y}} .
\end{aligned}
$$

\section{Nomenclature}

$a$ : Length of sandwich laminates

$b$ : Width of sandwich laminates

$c$ : Height of sandwich laminates

$c_{0}$ : Ambient speed of sound

$c_{1}$ : $\quad$ Speed of sound through the 3-D enclosure

$h=c$ :

$\frac{d}{d x}: \quad$ Differential operator

$E$ : $\quad$ Modulus of rigidity of the laminate

F: $\quad$ Applied end force amplitude

$x$ : Space coordinate along the length of the laminate

$y$ : $\quad$ Space coordinate along the width of the laminate

$z$ : $\quad$ Space coordinate along the height of the laminate

$\vec{I}: \quad$ Sound Intensity

$W: \quad$ Dynamic response

$\widetilde{W}: \quad$ Dynamic response in Laplace transform plane

$W^{F}$ : Dynamic response in Fourier transform plane

$\widetilde{W}^{F}: \quad$ Dynamic response in Fourier-Laplace transform plane

$\mu$ : $\quad$ Coefficient of friction at the interface of sandwich composite elastic beam

$\rho_{0}: \quad$ Ambient air density

$\rho=\rho_{0}{ }^{-1}$ :

$t$ : Time coordinate

$p_{0}$ : $\quad$ Clamping pressure at the interface

$\tau_{(x z)_{1}}$ : Shear stress at the upper half of the laminates interface

$\tau_{(x z)_{2}}: \quad$ Shear stress at the lower half of the laminates interface

\section{References}

[1] H. T. Banks, R. C. Smith, Y. Wang, D. E. Brown, V. L. Metcalf, and R. J. Silcox, "Noise control in a 3-D structural acoustic system: numerical and experimental implementation of a PDE-based methodology," in Proceedings of the 33rd IEEE 
Conference on Decision and Control, vol. 1, pp. 305-310, Lake Buena Vista, Fla, USA, December 1994.

[2] H. T. Banks, R. J. Silcox, and R. C. Smith, "The modeling and control of acoustic structure interaction problems via piezoceramic actuators: 2-D numerical examples," Journal of Vibration and Acoustics, vol. 116, no. 3, pp. 386-396, 1992.

[3] H. T. Banks, M. A. Demetriou, and R. C. Smith, "An $\mathrm{H}^{\infty} /$ MinMax periodic control in a two-dimensional structural acoustic model with piezoceramic actuators," IEEE Transactions on Automatic Control, vol. 41, no. 7, pp. 943-959, 1996.

[4] H. T. Banks, M. A. Demetriou, and R. C. Smith, " $\mathrm{H}^{\infty}$ control of noise in a 3-D structural acoustic system," in Proceedings of the IEEE Conference on Decision and Control, vol. 4, pp. 37193724, New Orleans, La, USA, December 1995.

[5] H. R. Pota, "Acoustical room transfer functions without using Green's functions," in Proceedings of the 40th IEEE Conference on Decision and Control (CDC '01), vol. 3, pp. 2588-2589, Orlando, Fla, USA, December 2001.

[6] J. D. Jones and C. R. Fuller, "Active control of structurallycoupled sound fields in elastic cylinders by vibrational force inputs," The International Journal of Analytical and Experimental Modal Analysis, vol. 5, no. 3, pp. 123-140, 1990.

[7] J. Pan, C. H. Hansen, and D. A. Bies, "Active control of noise transmission through a panel into a cavity-I: analytical Study," Journal of the Acoustical Society of America, vol. 87, no. 5, pp. 2098-2108, 1990.

[8] J. Pan and D. A. Bies, "The effect of fluid-structural coupling on sound waves in an enclosure-experimental part," Journal of the Acoustical Society of America, vol. 87, no. 2, pp. 708-717, 1990.

[9] S. Chen and Y. Liu, "A unified boundary element method for the analysis of sound and shell- like structure interactions. I. Formulation and verification," Journal of the Acoustical Society of America, vol. 106, no. 3, pp. 1247-1254, 1999.

[10] B. Fang, A. G. Kelkar, S. M. Joshi, and H. R. Pota, "Modelling, system identification, and control of acoustic-structure dynamics in 3-D enclosures," Control Engineering Practice, vol. 12, no. 8, pp. 989-1004, 2004.

[11] O. Damisa, V. O. S. Olunloyo, C. A. Osheku, and A. A. Oyediran, "Dynamic analysis of slip damping in clamped layered beams with non-uniform pressure distribution at the interface," Journal of Sound and Vibration, vol. 309, no. 3-5, pp. 349-374, 2008.

[12] V. O. S. Olunloyo, C. A. Osheku, and O. Damisa, "Vibration damping in structures with layered viscoelastic beam-plate," Journal of Vibration and Acoustics, vol. 130, no. 6, Article ID 061002, 26 pages, 2008.

[13] V. O. S. Olunloyo, O. Damisa, C. A. Osheku, and A. A. Oyediran, "Analysis of the effects of laminated depth and material properties on the damping associated with layered structures in a pressurized environment," Transaction of Applied Mechanics. In press.

[14] V. O. S. Olunloyo and C. A. Osheku, "On the analysis of the effects of thermal and time dependent interface pressure distribution on slip motion for sandwich elastic beams," in Proceedings of ASME International Mechanical Engineering Congress and Exposition (IMECE '07), vol. 10, pp. 763-774, Seattle, Wash, USA, November 2007, paper no. IMECE 200743896.

[15] V. O. S. Olunloyo, C. A. Osheku, and O. Damisa, "Slip damping with multilayered sandwich elastic beams," in Proceedings of ASME International Mechanical Engineering Congress and Exposition (IMECE '08), vol. 12, pp. 711-723, Boston, Mass, USA, October-November 2008, paper no. IMECE 2008-66245.
[16] L. D. Landau and E. M. Lifshitz, Fluid Mechanics, Course of Theoretical Physics, vol. 6, Institute of Physical Problems, USSR Academy of Science, Moscow, Russia, 2nd edition, 1987. 

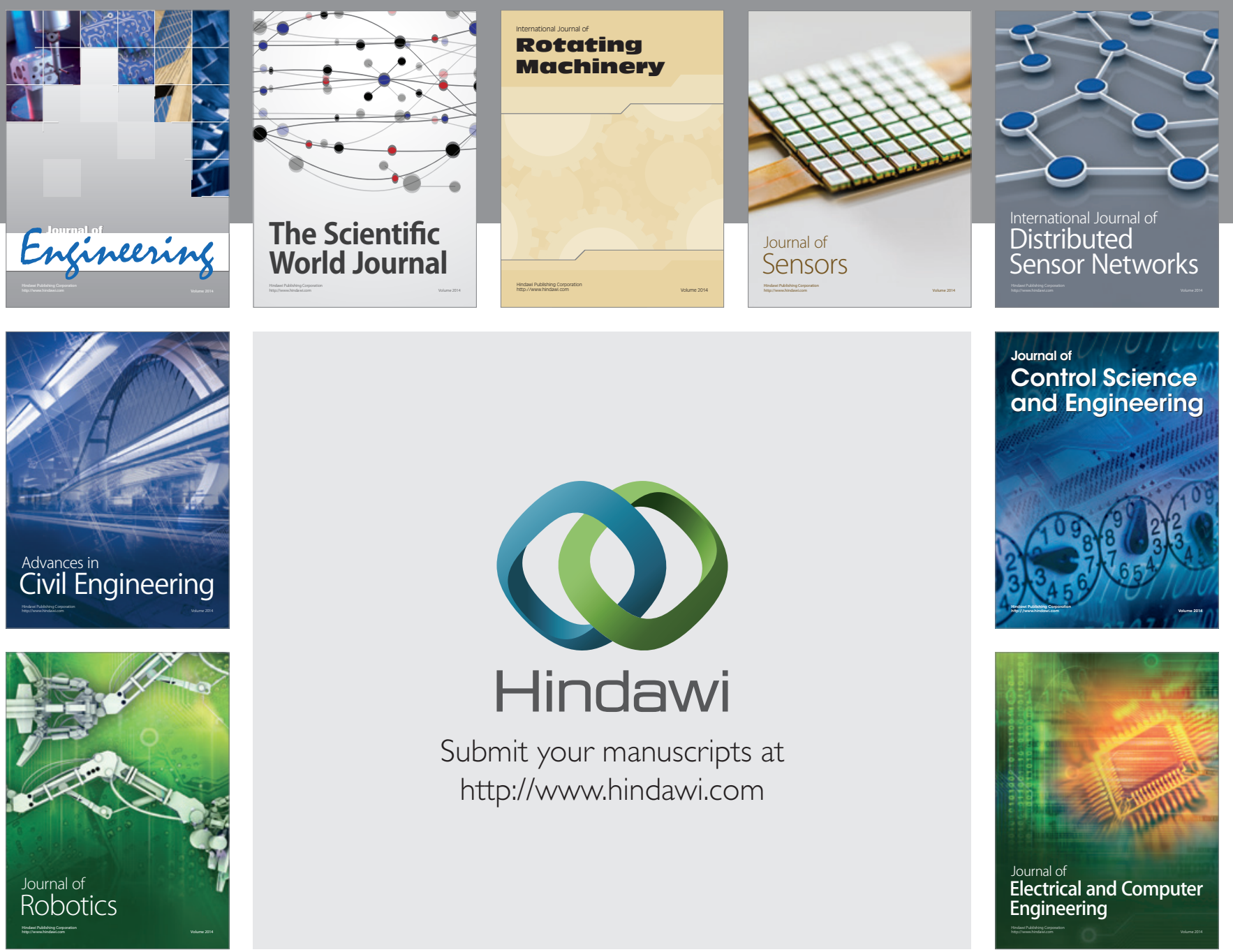

Submit your manuscripts at

http://www.hindawi.com
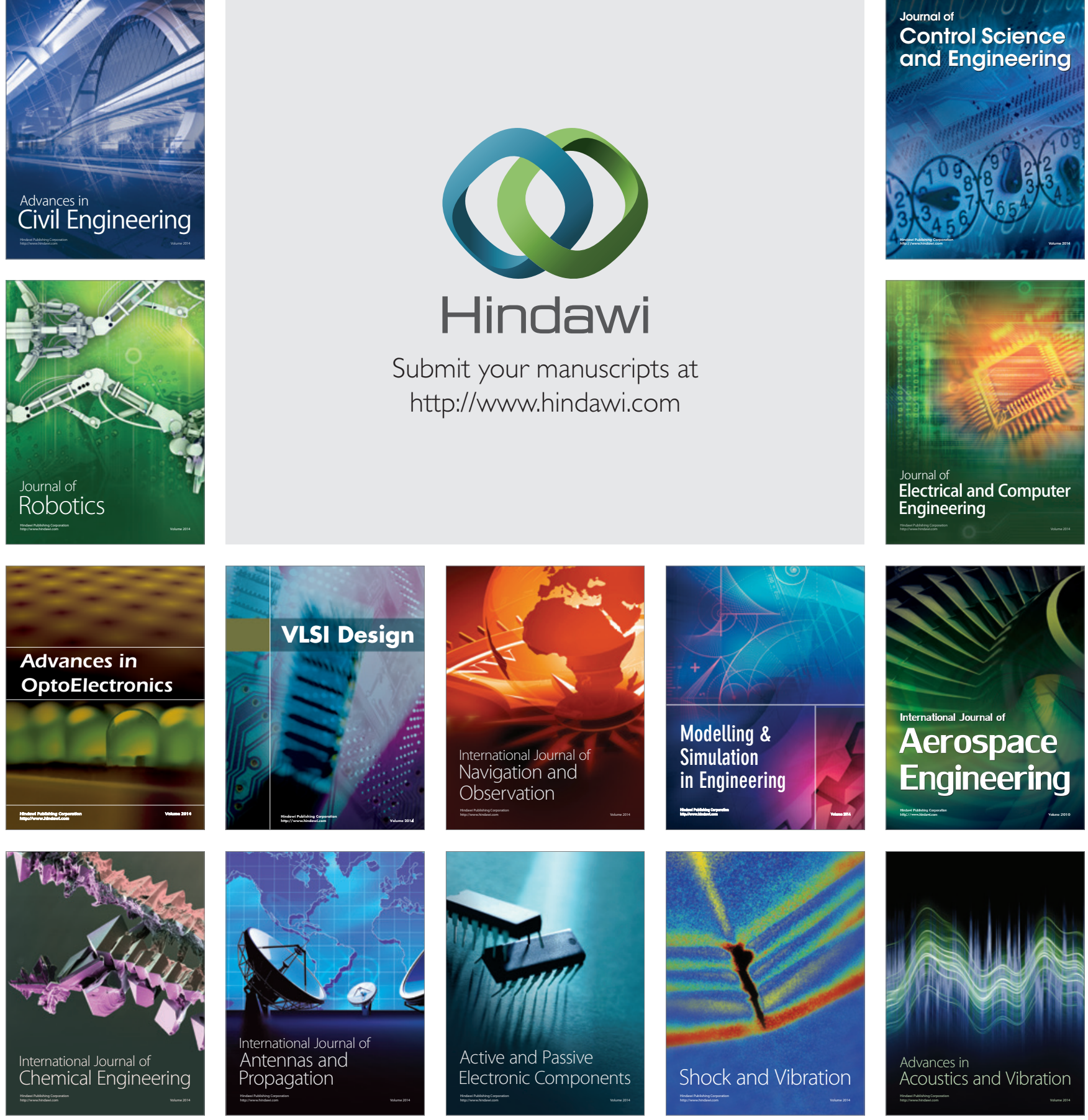